\title{
LINK MAPS IN ARBITRARY MANIFOLDS AND THEIR HOMOTOPY INVARIANTS
}

\author{
ULRICH KOSCHORKE *
}

FB Mathematik, Universität Siegen, D-57068 Siegen

\begin{abstract}
In this paper we generalize Milnor's $\mu$-invariants of classical links to certain (" $\kappa$-Brunnian") higher dimensional link maps into fairly arbitrary manifolds. Our approach involves the homotopy theory of configuration spaces and of wedges of spheres. We discuss the strength of these invariants and their compatibilities e.g. with (Hilton decompositions of) linking coefficients. Our results suggest, in particular, a conjecture about possible new link homotopies.
\end{abstract}

\section{Introduction}

Given $r \geq 2$ and arbitrary dimensions $p_{1}, \ldots, p_{r} \geq 1$ and $m \geq 3$, we want to study link maps

$$
f=f_{1} \amalg \cdots \amalg f_{r}: S^{p_{1}} \amalg \cdots \amalg S^{p_{r}} \longrightarrow M
$$

(i.e. the spheres $S^{p_{j}}$ have pairwise disjoint images under the continuous maps $f_{j}, 1 \leq j \leq r$ ) up to link homotopy (i.e. up to continuous deformations through such link maps). Here $M$ denotes a smooth $m$-dimensional manifold which we assume (without much loss of generality) to be also connected and to have no boundary. One further restriction seems to be more essential for our approach: $M$ must satisfy a certain openness condition (see 2.1 below); in particular, closed manifolds must be punctured first before we can apply certain parts of our theory (cf. 5.3 below).

Our basic invariants are the homotopy class (in the standard sense)

$$
\kappa(f):=[\widehat{f}] \in\left[S^{p_{1}} \times \cdots \times S^{p_{r}}, \widetilde{C}_{r}(M)\right]
$$

and its base point preserving analogon

$$
\kappa^{b}(f):=[\widehat{f}] \in\left[\left(S^{p_{1}} \times \cdots \times S^{p_{r}}, *\right),\left(\widetilde{C}_{r}(M),\left(y_{1}^{\circ}, \ldots, y_{r}^{\circ}\right)\right)\right]
$$

* Supported in part by the Brazilian-German CNPq-GMD agreement 
where the product map $\widehat{f}=f_{1} \times \cdots \times f_{r}$ takes values in the configuration space of ordered $r$-tuples of pairwise distinct points in $M$. Now it is a very special feature of this product map that the $j$ th component of the value $\widehat{f}\left(x_{1}, \ldots, x_{r}\right)$ depends only on the $j$ th component $x_{j}$ of the argument. In particular, any link map and - likewise - any link homotopy can be reconstructed from its product map.

By the transition to standard homotopy this "equivariance" gets lost. Thus at first sight our $\kappa$-invariants seem to be rather crude. Yet surprisingly in many interesting cases they turn out to determine link maps completely up to link homotopy.

The special symmetry properties of $\widehat{f}$ suggest also that the $\kappa$-invariants may lie in much smaller and more manageable homotopy sets (or even groups). This is indeed the case if we concentrate on highest order linking phenomena and assume that $f$ is $\kappa$-Brunnian (i.e. $\widehat{f}$ is nulhomotopic when restricted to the complement of the top cell in $\left.S^{p_{1}} \times \cdots \times S^{p_{r}}\right)$. Then we can simplify e.g. $\kappa^{b}(f)$ until we arrive at the following situation

$$
\begin{array}{ccc}
\kappa^{\prime}(f) & \longrightarrow & \mu(f)=\left\{\mu_{\gamma}(f)\right\}_{\gamma \in \Sigma_{r-2}} \\
\in & \in \\
\pi_{|p|}^{\prime}\left(M-\left\{y_{1}^{\circ}, \ldots, y_{r-1}^{\circ}\right\}\right) \stackrel{h \circ c_{a}}{\longrightarrow} \underset{\gamma \in \Sigma_{r-2}}{\bigoplus} \pi_{|p|-(r-1)(m-2)-1}^{S},
\end{array}
$$

where

$$
|p|:=p_{1}+\cdots+p_{r}
$$

Here $\kappa^{\prime}(f)$ is a well defined invariant which contains precisely as much information as $\kappa^{b}(f)$; it lies in a certain ("reduced") subgroup of the homotopy group of $M$, punctured at $r-1$ points. $h$ denotes a Hopf homomorphism which is known to be bijective if $|p| \leq r(m-2)$ (cf. [K 5], § 3). In the same dimension range the homomorphism $c_{a}$ performs some kind of localization: it forgets everything which occurs outside of a suitably embedded ball in $M$. In particular, our total $\mu$-invariant $\mu(f):=h \circ c_{a}\left(\kappa^{\prime}(f)\right.$ ) (whose components are indexed by the permutations $\gamma$ of $r-2$ elements) does not capture phenomena related to the fundamental group $G$ of $M$. To make up for this, we introduce the stronger invariant $\widetilde{\mu}(f)$ which often turns out to consist of the $\mu$-invariants of all possible liftings of $f$ to the universal covering space $\widetilde{M}$ of $M$ (see definition 2.11 and corollary 2.16 below).

QUESTION. How much information gets lost by the simplification procedure which extracts $\widetilde{\mu}(f)$ from $\kappa^{b}(f)$ ?

We encounter two sharply different situations.

CASE I: $\widetilde{M}$ is $(m-2)$-connected. Here the answer is "none" at least if $p_{1}, \ldots, p_{r}$ $\leq m-2$ : we may replace the unwieldy invariant $\kappa^{b}(f)$ by the much simpler "numerical" invariant $\widetilde{\mu}(f)$ which is seen to contain precisely as much information (see theorem 5.6).

This is illustrated e.g. in the classical dimension setting when $p_{1}, \ldots, p_{r}=$ $1, m=3$ and $M$ is oriented. Here $\widetilde{\mu}$ takes values in the group ring of $G^{r-1} \times \Sigma_{r-2}$ and can be proved to describe just (a reparametrized version of) the "elementary sentence" which J. Milnor [M 1] attached to $\kappa$-Brunnian links (he would call them almost trivial). Thus Milnor's results (obtained by an algebraic 
analysis of link groups) can be reinterpreted to show that $\widetilde{\mu}$, and hence $\kappa^{b}$, determines $\kappa$-Brunnian link maps fully up to link homotopy. Similarly, we can rephrase work of U. Dahlmeier as an injectivity result for $\kappa$ on all link maps with two components in the 3 -dimensional solid torus or punctured projective space. (All this can be found in $\S 6$ ).

CASE II: $\widetilde{M}$ is not $(m-2)$-connected. Here $\widetilde{\mu}$ is often definitely weaker than $\kappa^{b}$.

To get a large supply of examples, we make a detailed study (in $\S 4$ ) of link homotopy classes of the form $e_{*}\left(f_{r}\right)$ which are described by $r-1$ standard spheres in parallel hyperplanes in a suitable small ball $B \subset M$ and by a "linking coefficient"

$$
\left[f_{r}\right] \in \pi_{p_{r}}^{\prime}\left((M-\stackrel{\circ}{B}) \vee \bigvee_{j=1}^{r-1} S_{j}^{m-p_{j}-1}\right)
$$

involving the meridians $S_{j}^{m-p_{j}-1}$. Such link homotopy classes are often provided by homotopy Brunnian links in codimensions $>2$ (cf. 4.6). A careful analysis comparing the "input" $f_{r}$ to the resulting invariant ("output") $\kappa^{\prime}\left(e_{*}\left(f_{r}\right)\right)$ shows that we obtain a group homomorphism

$$
\kappa^{\prime} \circ e_{*}: \pi_{p_{r}}^{\prime}\left(M \vee \bigvee_{j=1}^{r-1} S_{j}^{m-p_{j}-1}\right) \longrightarrow \pi_{|p|}^{\prime}\left(M \vee \bigvee_{j=1}^{r-1} S^{m-1}\right)
$$

which is compatible with Whitehead products or, in other words, with higher dimensional commutators (see theorem 4.10). Thus, frequently $\kappa^{\prime} \circ e_{*}$ can be expressed entirely in terms of suspension homomorphisms which are defined on homotopy groups of spheres. As a consequence one can easily exhibit settings where $\kappa$ and $\kappa^{\prime}$ classify link maps fully up to link homotopy or where the $\kappa^{\prime}$-or $\widetilde{\mu}$-invariants can realize all values in their range. In particular, neither $\kappa^{\prime}$ nor $\mu$ or $\widetilde{\mu}$ can be accomodated in general in smaller groups than the ones indicated in their definition (cf. I.1 and 2.6): no further universal restrictions or relations follow from the equivariance of $\kappa_{M}^{b}$; this insight supplements very nicely our effort to make the range of our invariants as small and simple as possible. Furthermore, often a link map $f$ exists with $\widetilde{\mu}(f)=0$ but $\kappa^{\prime}(f) \neq 0$ (see e.g. 4.11 or 5.9). The vanishing result in theorem 4.10 gives also a (potentially decisive) hint for where to look for new link nulhomotopies (compare 4.12 and 4.13).

Throughout this paper we study our invariants in two parallel settings. It is technically convenient to discuss them first in the setting of base point preserving link maps and homotopies. Then we analyse (e.g. in $\S 3$ ) the transition to base point free link homotopy theory which seems geometrically more natural. If $M$ is 1-connected, the two versions of our theory coincide.

Since closed manifolds must be punctured before we can apply our approach fruitfully (for an example see 1.6; compare also 5.3), we discuss also in some detail the effect of removing a discrete subset from $M$ (cf. 5.1 and 5.2). Moreover, in very general situations we relate our invariants to "linking coefficients" (cf. 2.20).

Acknowledgement. It is a pleasure to thank Derek Hacon, Uwe Kaiser and Karl Otto Stöhr for stimulating discussions. 
Notations and Conventions. Spheres, their products, wedges etc. are endowed with a base point $*$ (and hence also with a cell decomposition); in a wedge $*$ is the wedge point. The configuration spaces $\widetilde{C}_{s}(M)$ and $\widetilde{C}_{s}(\widetilde{M})$ of $M$ and of its universal covering $\widetilde{M}$ are endowed with a choice of base points $y^{\circ}=$ $\left(y_{1}^{\circ}, \ldots, y_{s}^{\circ}\right)$ and $\widetilde{y}^{\circ}=\left(\widetilde{y}_{1}^{\circ}, \ldots, \widetilde{y}_{s}^{\circ}\right)$ compatible with the obvious projections; $G \cong$ $\pi_{1}(M)$ denotes the group of covering transformations of $\widetilde{M}$. We write $\Sigma_{s}$ for the permutation group of $s$ elements. For any $r$-tuple $(p)=\left(p_{1}, \ldots, p_{r}\right)$ of dimensions we put $|p|=p_{1}+\cdots+p_{r}$. All manifolds are assumed to be Hausdorff spaces.

Persistent use of Pontryagin-Thom bijections is understood. E.g. homotopy classes into a wedge of spheres are considered as bordism classes of framed links and vice versa. Similarly, $\pi_{*}^{S} \cong \Omega_{*}^{f r}$ denotes framed (i.e. stably parallelized) bordism.

From now on we write $\kappa^{\prime}(f)$ for the invariant which was denoted by $\widetilde{\kappa}(f)$ in the paper [K 5] where covering spaces played no role.

\section{$\S$ 1. The $\kappa$-invariants}

In this section we introduce our basic link homotopy invariants $\kappa(f)$ and $\kappa^{b}(f)$.

Let $M$ be any topological space. For an integer $r \geq 1$ let

$$
\widetilde{C}_{r}(M):=\left\{\left(y_{1}, \ldots, y_{r}\right) \in M^{r} \mid y_{i} \neq y_{j} \text { for } i \neq j, \quad 1 \leq i, j \leq r\right\}
$$

denote the (ordered) configuration space of $r$ pairwise distinct points in $M$ (with the subspace topology inherited from $M^{r}$ ).

Given an $r$-tuple $(p)=\left(p_{1}, \ldots, p_{r}\right)$ of nonnegative integers, we denote by $\amalg S^{p_{j}}$ and

$$
S^{(p)}:=S^{p_{1}} \times \cdots \times S^{p_{r}}
$$

the disjoint union and product, resp., of the corresponding unit spheres.

Definition 1.3. A continuous map

$$
f=f_{1} \amalg \cdots \amalg f_{r}: \amalg S^{p_{j}} \longrightarrow M
$$

is called a link map if the component maps $f_{j}$ have pairwise disjoint images.

This is equivalent to the following condition: the product map

$$
\widehat{f}:=f_{1} \times \cdots \times f_{r}
$$

maps $S^{(p)}$ into the subspace $\widetilde{C}_{r}(M)$ of $M^{r}$. Similarly a link homotopy of $f$ (i.e. a homotopy through link maps) gives rise to a homotopy (in the usual sense) in $\widetilde{C}_{r}(M)$. Thus we obtain the link homotopy invariant

$$
\kappa(f)=\kappa_{M}(f):=[\widehat{f}] \in\left[S^{(p)}, \widetilde{C}_{r}(M)\right]
$$

in the base point free standard homotopy set to the right. (We will often drop the subscript $M$ if no confusion can arise). 
Example 1.6 : open vs. closed manifolds. Let $r=2$.

If $M=\mathbb{R}^{m}$, then $\widetilde{C}_{2}(M)$ is homotopy equivalent to $S^{m-1}$ (via $\left(y_{1}, y_{2}\right) \longrightarrow$ $\left.\left(y_{1}-y_{2}\right) /\left\|y_{1}-y_{2}\right\|\right)$; the degree (or suspension) map $\left[S^{(p)}, S^{m-1}\right] \longrightarrow \Omega_{*}^{f r} \cong \pi_{*}^{S}$ takes $\kappa_{\mathbb{R}} m(f)$ to the generalized linking number $\pm \alpha(f)$ which is known often to capture much of the linking behaviour of $f$ (see e.g. [K 2]) and to classify $f$ completely up to link homotopy if $2 p_{1}+2 p_{2} \leq 3 m-5$ (see [HK]).

If $M=S^{m}$, then the projection

$$
p r: \widetilde{C}_{2}\left(S^{m}\right) \longrightarrow \widetilde{C}_{1}\left(S^{m}\right)=S^{m},
$$

$\operatorname{pr}\left(y_{1}, y_{2}\right)=y_{1}$, is a homotopy equivalence (its fiber being contractible) and compatible with $\widehat{f}$ and with the first projection in $S^{(p)}$; since also $f_{1}$ is nulhomotopic in $S^{m}-f_{2}$ (point), the invariant $\kappa_{S^{m}}(f)$ is trivial.

Definition 1.7. A link map $f$ (as in 1.3) is called homotopy trivial if it is link homotopic to a link map all of whose component maps are constant.

$f$ is called homotopy Brunnian if every proper sublink map is trivial in this sense.

$f$ is called $\kappa_{M}$-trivial (or simply $\kappa$-trivial) if $\kappa_{M}(f)$ is trivial, i.e. $\widehat{f}$ is nulhomotopic (in the standard base point free sense).

$f$ is called $\kappa_{M}$-Brunnian (or simply $\kappa$-Brunnian) if for $j=1, \ldots, r$ the product map $\widehat{f}$ is nulhomotopic when restricted to

$$
X_{j}:=S^{p_{1}} \times \cdots \times S^{p_{j-1}} \times *_{j} \times S^{p_{j+1}} \times \cdots \times S^{p_{r}}
$$

(where $*_{j} \in S^{p_{j}}$ is the base point).

It will be useful to study also the base point preserving version of the $\kappa$-invariant. Fix a base point $y^{\circ}=\left(y_{1}^{\circ}, \ldots, y_{r}^{\circ}\right)$ of $\widetilde{C}_{r}(M)$. If the link map $f$ (as in 1.3) preserves base points (i.e. $f_{j}\left(*_{j}\right)=y_{j}^{\circ}$ for $j=1, \ldots, r$ ), then the homotopy class (of pointed spaces)

$$
\kappa^{b}(f)=\kappa_{M}^{b}(f):=[\widehat{f}] \in\left[\left(S^{(p)}, *\right),\left(\widetilde{C}_{r}(M), y^{\circ}\right)\right]
$$

is obviously an invariant of the base point preserving link homotopy class of $f$.

\section{$\S 2$. The $\mu$ - and $\widetilde{\mu}$-invariants of a base point preserving $\kappa-$ Brunnian link map}

From now on we assume that $r \geq 2, p_{1}, \ldots, p_{r} \geq 1$ and that $M$ is a smooth connected manifold, without boundary, of dimension $m \geq 3$, having a countable basis and satisfying the following ("openness")

Condition 2.1. There exists a closed subset $A$ of $M$ which is diffeomorphic to $\mathbb{R}^{m-1} \times[0, \infty)$ (or, equivalently, to the halfball $\left\{x \in \mathbb{R}^{m} \mid\|x\| \leq 1, x_{m}>0\right\}$ ).

This condition holds e.g. if $M$ is the interior $\bar{M}-\partial \bar{M}$ of any smooth $m^{-}$ manifold $\bar{M}$ with nonempty boundary $\partial \bar{M}$, or if $M$ is obtained by puncturing any smooth $m$-manifold without boundary (i.e. by removing one point).

It follows in particular from 2.1 that there is a smooth embedding ("arc")

$$
a:[0, \infty) \hookrightarrow M
$$


which is proper (i.e. the inverse image of any compact subset of $M$ is again compact). This implies e.g. that the locally trivial fibration (cf. [FN])

$$
p r: \widetilde{C}_{r}(M) \longrightarrow \widetilde{C}_{r-1}(M)
$$

$\operatorname{pr}\left(y_{1}, \ldots, y_{r}\right):=\left(y_{1}, \ldots, y_{r-1}\right)$, admits a continuous section (just add a "faraway point" $y_{r}=a(t)$ with $t \gg 0$ depending continuously on $y_{1}, \ldots, y_{r-1}$; e.g. $y_{r}=$ $\left(0, \sum_{i=1}^{r-1} \varphi\left(y_{i}\right)+1\right) \in \mathbb{R}^{m-1} \times[0, \infty) \cong A$ where $\varphi$ is the projection to the last coordinate on $A$ and $\varphi \equiv 0$ outside of $A$ ). Therefore, the fiber inclusion

$$
\text { incl }: M-\left\{y_{1}^{\circ}, \ldots, y_{r-1}^{\circ}\right\} \hookrightarrow \widetilde{C}_{r}(M)
$$

induces a monomorphism of homotopy groups.

Now consider a link map $f$ as in definition 1.3 which, in addition, preserves base points (compare 1.8). We want to concentrate on linking phenomena of highest order and therefore assume also that $f$ is $\kappa_{M}$-Brunnian (cf. 1.7).

Then we can conclude that there is a unique element

$$
\kappa^{\prime}(f)=\kappa_{M}^{\prime}(f) \in \pi_{|p|}^{\prime}\left(M-\left\{y_{1}^{\circ}, \ldots, y_{r-1}^{\circ}\right\}, y_{r}^{\circ}\right):=\bigcap_{j=1}^{r-1} \operatorname{ker}\left(\operatorname{fill}_{j *}\right)
$$

which gets mapped to $\kappa_{M}^{b}(f)$ under the injective composite map

$$
\pi_{|p|}\left(M-\left\{y_{1}^{\circ}, \ldots, y_{r-1}^{\circ}\right\}, y_{r}^{\circ}\right) \stackrel{\text { incl }_{*}}{\hookrightarrow} \pi_{|p|}\left(\widetilde{C}_{r}(M), y^{\circ}\right) \stackrel{\text { quot* }^{*}}{\hookrightarrow}\left[\left(S^{(p)}, *\right),\left(\widetilde{C}_{r}(M), y^{\circ}\right)\right]
$$

(compare $[\mathrm{P}]$, Satz 12, 13, and 20, as well as $[\mathrm{K} 5]$, $\S 4$ ). Here fill $j$ denotes the inclusion into $M-\left\{y_{1}^{\circ}, \ldots, y_{j-1}^{\circ}, y_{j+1}^{\circ}, \ldots, y_{r-1}^{\circ}\right\}$ ("filling back in" the missing point $\left.y_{j}^{\circ}\right)$ and quot $: S^{(p)} \longrightarrow S^{|p|}$ pinches all but the top cell into a point; moreover, $|p|:=p_{1}+\cdots+p_{r}$.

In order to extract first $\mu(f)$ and then also $\widetilde{\mu}(f)$ from $\kappa^{\prime}(f)$, consider the following diagram.

Here the reduced homotopy group

$$
\pi_{|p|}^{\prime}\left(\bigvee^{r-1} S^{m-1}\right):=\bigcap_{j=1}^{r-1} \operatorname{ker}\left(\pi_{|p|}\left(\bigvee_{i=1}^{r-1} S_{i}^{m-1}, *\right) \longrightarrow \pi_{|p|}\left(\bigvee_{\substack{i=1 \\ i \neq j}}^{r-1} S_{i}^{m-1}, *\right)\right)
$$


is defined in analogy to (2.4); the Hopf homomorphism $h_{\gamma}$ measures (up to framed bordism) that part of the overcrossing locus of a framed link where the components are "stacked on top of one another" according to the order given by the permutation $\gamma \in \Sigma_{r-2}$ (with the $(r-1)$ st link component lying at the bottom; cf. [K 5], § 3).

The construction of the homomorphism $c_{a}$ depends on the choice $a=\left(a_{1}, \ldots\right.$, $\left.a_{r-1}\right)$ of proper, smoothly embedded and pairwise disjoint arcs $a_{j}:[0, \infty) \hookrightarrow$ $M-\left\{y_{r}^{\circ}\right\}$ such that $a_{j}(0)=y_{j}^{\circ}$, together with local orientations of $M$ at $y_{j}^{\circ}$ (which yield normal orientations and - unique up to isotopy - framings of $a_{j}$ in $M), j=1, \ldots, r-1$; such arcs exist due to condition 2.1'. Given a generic smooth map $u:\left(S^{|p|}, *\right) \longrightarrow\left(M-\left\{y_{1}^{\circ}, \ldots, y_{r-1}^{\circ}\right\}, y_{r}^{\circ}\right), \quad c_{a}([u])$ is defined by the framed bordism class of the link

$$
\coprod_{j=1}^{r-1} u^{-1}\left(a_{j}[0, \infty)\right) \quad \subset \quad S^{|p|}-\{*\} \cong \mathbb{R}^{|p|} .
$$

Remark 2.8. $h_{\circ} c_{a}$ is still well defined when the $a_{j}$ are only (possibly singular) proper disjoint paths (and hence, in general, the "intersections" $u \pitchfork a_{j}=$ $\left(u \times a_{j}\right)^{-1}$ (diagonal $\left.\Delta \subset M \times M\right)$ define only a nonembedded nonspherical link map into $\mathbb{R}^{|p|}$; they are stably framed since the obvious homotopy $a_{j} \sim y_{j}^{\circ}$ induces a trivialization of the pullback of the normal bundle $\nu(\Delta, M \times M) \cong \operatorname{proj}_{2}^{*}(T M)$ under $u \times a_{j}$ ). In particular, $h \circ c_{a}$ remains invariant under proper disjoint homotopies of the $a_{j}$.

We define the $\mu$-invariants of the $\kappa_{M}$-Brunnian link map $f$ by

$$
\mu_{\gamma}(f)=\mu_{M, \gamma}(f):=h_{\gamma} \circ c_{a}\left(\kappa_{M}^{\prime}(f)\right) \in \pi_{|p|-(r-1)(m-2)-1}^{S}, \quad \gamma \in \Sigma_{r-2} .
$$

Together they yield the total $\mu$-invariant of $f$

$$
\mu(f)=\mu_{M}(f):=\left\{\mu_{M, \gamma}(f)\right\} \in \bigoplus_{\gamma \in \Sigma_{r-2}} \pi_{|p|-(r-1)(m-2)-1}^{S} .
$$

Note that a change of our chosen local orientations of $M$ at $k$ points $y_{j}^{\circ}$ just modifies $h \circ c_{a}$ and hence $\mu(f)$ by the factor $(-1)^{k}$.

Next we use the universal covering $p: \widetilde{M} \longrightarrow M$ to refine the $\mu$-invariant. Fix a base point $\widetilde{y}^{\circ}=\left(\widetilde{y}_{1}^{\circ}, \ldots, \widetilde{y}_{r}^{\circ}\right)$ of $\widetilde{C}_{r}(\widetilde{M})$ which gets mapped to $y^{\circ} \in \widetilde{C}_{r}(M)$ by $p$. Choose a fixed global orientation of $\widetilde{M}$ which then also determines all our choices of local orientations of $\widetilde{M}$. Let

$$
\widetilde{\operatorname{fill}}_{j *}: \pi_{|p|}\left(\widetilde{M}-\bigcup_{i=1}^{r-1} p^{-1}\left\{y_{i}^{\circ}\right\}, \widetilde{y}_{r}^{\circ}\right) \longrightarrow \pi_{|p|}\left(\widetilde{M}-\bigcup_{\substack{i=1 \\ i \neq j}}^{r-1} p^{-1}\left\{y_{i}^{\circ}\right\}, \widetilde{y}_{r}^{\circ}\right)
$$

be induced by the inclusion (filling back in the fiber $p^{-1}\left\{y_{j}^{\circ}\right\}$ of the covering map). Our $\widetilde{\mu}^{-}$invariants are constructed from the unique element

$$
\widetilde{\kappa}^{\prime}(f)=\widetilde{\kappa}_{M}^{\prime}(f) \in \pi_{|p|}^{\prime}\left(\widetilde{M}-\bigcup_{j=1}^{r-1} p^{-1}\left\{y_{j}^{\circ}\right\}, \widetilde{y}_{r}^{\circ}\right):=\bigcap_{j=1}^{r-1} \operatorname{ker}\left(\widetilde{\operatorname{fill}}_{j *}\right)
$$


such that $p_{*}\left(\widetilde{\kappa}^{\prime}(f)\right)=\kappa^{\prime}(f)$ (see diagram 2.6).

Let $G \cong \pi_{1}\left(M ; y_{r}^{\circ}\right)$ denote the group of covering transformations. Each $g=$ $\left(g_{1}, \ldots, g_{r-1}\right) \in G^{r-1}$ determines the system $\left(g_{1} \circ \widetilde{a}_{1}, \ldots, g_{r-1} \circ \widetilde{a}_{r-1}\right)$ of proper disjoint $\operatorname{arcs}$ in $\widetilde{M}$ which lift the chosen $\operatorname{arcs} a_{1}, \ldots, a_{r-1}$ (given in $M$ ) such that $g_{j} \circ \widetilde{a}_{j}(0)=g_{j}\left(\widetilde{y}_{j}^{\circ}\right)$ for $1 \leq j<r$. Let $\widetilde{c}_{a}^{g}$ be the resulting homomorphism (constructed as in 2.7). This describes the remaining arrows in diagram 2.6.

We define the (total) $\widetilde{\mu}$-invariant of the base point preserving $\kappa_{M}$-Brunnian link map $f$ by

$$
\widetilde{\mu}(f)=\widetilde{\mu}_{M}(f):=\left\{\widetilde{\mu}_{M, g}(f)\right\} \in \bigoplus_{(g, \gamma) \in G^{r-1} \times \Sigma_{r-2}} \pi_{|p|-(r-1)(m-2)-1}^{S}
$$

where $\widetilde{\mu}_{M, g}(f):=h \circ \widetilde{c}_{a}^{g}\left(\widetilde{\kappa}_{M}^{\prime}(f)\right)$.

Now let $k$ denote the number of ("base") points $y_{j}^{\circ}, \quad 1 \leq j \leq r-1$, where the chosen local orientation of $M$ is not given by the orientation of $\widetilde{M}$ at $\widetilde{y}_{j}^{\circ}$ via the covering map $p$. For $g=\left(g_{1}, \ldots, g_{r-1}\right) \in G^{r-1} \operatorname{put} \operatorname{sign}(g)=\prod_{j} \operatorname{sign}\left(g_{j}\right)$ where $\operatorname{sign}\left(g_{j}\right)$ equals +1 (or -1 ) when $g_{j}$ is orientation preserving (or reversing, resp.), $j=1, \ldots, r-1$.

\section{Proposition 2.12.}

$$
\mu_{M}(f)=(-1)^{k} \sum_{g \in G^{r-1}} \operatorname{sign}(g) \widetilde{\mu}_{M, g}(f)
$$

Proof. Represent $\kappa_{M}^{\prime}(f)$ by a generic smooth map $u:\left(S^{|p|}, *\right) \longrightarrow\left(M-\left\{y_{1}^{\circ}, \ldots\right.\right.$, $\left.y_{r-1}^{\circ}\right\}, y_{r}^{\circ}$ ) and let $\widetilde{u}$ be the lifting to $\widetilde{M}$ such that $\widetilde{u}(*)=\widetilde{y}_{r}^{\circ}$. Then, in 2.7, $u^{-1}\left(a_{j}[0, \infty)\right)$ equals the inverse image $\bigcup \widetilde{u}^{-1}\left(g_{j} \circ \widetilde{a}_{j}[0, \infty)\right)$ of all liftings $g_{j} \circ \widetilde{a}_{j}, g_{j} \in G$, of the arc $a_{j}$. For every $\gamma \in \Sigma_{r-2}$ this leads to the desired decomposition

$$
h_{\gamma}\left(\amalg u^{-1}\left(a_{j}[0, \infty)\right)\right)=\sum_{g \in G^{r-1}} \pm h_{\gamma}\left(\amalg \widetilde{u}^{-1}\left(g_{j} \circ \widetilde{a}_{j}[0, \infty)\right)\right)
$$

of $\mu_{\gamma}(f)=h_{\gamma} \circ c_{a}[u]$ into a finite sum.

Example 2.13. If $M$ is simply connected, then $\mu_{M}(f)= \pm \widetilde{\mu}_{M}(f)$ is invariant under base point free link homotopies of $f$ (since $\kappa_{M}^{b}(f)$ is). If in addition $M$ is contractible, then there exists a (homology and hence weak) homotopy equivalence $\vee^{r-1} S^{m_{1}} \hookrightarrow M-\left\{y_{1}^{\circ}, \ldots, y_{r-1}^{\circ}\right\}$ such that the induced isomorphism is an inverse of $c_{a}$ (cf. diagram 2.6; compare also the proof of theorem 5.6 below for a similar argument); therefore in this case the $\mu$-invariant of $f$ is also independent of the choice of the arcs involved in its construction. In particular, if $M=\mathbb{R}^{m}$ we obtain again the well defined $\mu$-invariants which were studied in [K 5] and which generalize Milnor's $\mu$-invariants of classical almost trivial links.

Example 2.14: $r=2, M \subset \mathbb{R}^{m}$ open. Given a singular manifold $u$ in $M-\left\{y_{1}^{\circ}\right\}$, its intersection in $\mathbb{R}^{m}$ with a ray $y_{1}^{\circ}+[0, \infty) \cdot z\left(z \in S^{m-1}\right)$ is bordant to its intersection with a suitable arc $a_{1}$ in $M$ (as in 2.7 or 2.8). Therefore, if the value of $\mu_{M}(f)$ is independent of the choice of the arc $a_{1}$ (e.g. if $p_{1}+p_{2} \leq 2 m-4$, cf. 
the proposition 2.15 below), then this invariant coincides with the framed bordism class of the inverse image $\varphi_{f}^{-1}\{z\}$ of $z$ under (a suitable approximation of) the map

$$
\varphi_{f}: S^{p_{1}} \times S^{p_{2}} \longrightarrow S^{m-1},
$$

$\varphi_{f}\left(x_{1}, x_{2}\right):=\left(f_{1}\left(x_{1}\right)-f_{2}\left(x_{2}\right)\right) /\left\|f_{1}\left(x_{1}\right)-f_{2}\left(x_{2}\right)\right\|$, i.e. with the " $\alpha$-invariant" $\mu_{\mathbb{R}^{m}}(f)$ (compare e.g. [K 1 ]) which is always well defined (whether $f$ is $\kappa_{M^{-}}$ Brunnian or not).

It remains to study in general the impact of the choices of the arcs which are used in the construction of $c_{a}$ or $\widetilde{c}_{a}^{g}$ (see also remark 2.8).

Proposition 2.15. Assume $|p| \leq r(m-2)$.

Then the homomorphisms $c_{a}$ and $\widetilde{c}_{a}^{g}, g \in G^{r-1}$, in diagram 2.6 are independent of the choice of the system of arcs a involved in their construction. In particular, the invariants $\mu(f)$ and $\widetilde{\mu}(f)$ depend only on the base point preserving link homotopy class of $f$ (and on the choice of (local) orientations).

Moreover, the homomorphisms $h$ and $\widetilde{h}$ in diagram 2.6 are bijective.

Proof. For the last claim see theorem 3.1 in [K 5].

To prove the first statement consider two systems $a, a^{\prime}$ of arcs and a map $u$ as in 2.7. Since suitable proper homotopies do not affect $h \circ c_{a}$ or $h \circ c_{a^{\prime}}$ we can make all arcs in $a$ and $a^{\prime}$ disjoint (except at their starting points). So it suffices to check what happens when we substitute just one arc $a_{j}$ at a time by $a_{j}^{\prime}$, e.g. for $j=1$.

Let $a_{0}: \mathbb{R} \hookrightarrow M-\bigcup^{r-1} a_{j}([0, \infty))$ be a smooth proper path which first follows a parallel of $a_{1}$ (but in the opposite direction) until it reaches a neighbourhood of $a_{1}(0)=a_{1}^{\prime}(0)=y_{1}^{\circ}$ and then proceeds along $a_{1}^{\prime}$. The corresponding inverse image $u^{-1}\left(a_{0}(\mathbb{R})\right)$ adds a new disjoint component to the framed link in 2.7. Equivalently, it defines an extended homotopy class

$$
v \in \pi_{|p|}\left(S_{0}^{m-1} \vee \bigvee_{j=1}^{r-1} S_{j}^{m-1}\right)
$$

which maps to $c_{a}[u]$ (or $c_{\left(a_{1}^{\prime}, a_{2}, \ldots, a_{r-1}\right)}[u]$, resp.) when $S_{0}^{m-1}$ collapses to a point (or maps identically to $S_{1}^{m-1}$, resp.) while $\bigvee^{r-1} S_{j}^{m-1}$ remains unchanged. (In order to understand the second case, deform the arc $a_{1}^{\prime}$ until it first follows $a_{1}$ beyond the compact image of $u$ and then returns back along $a_{0}$ ).

If there is a nulhomotopy of $u$ in $M-\left\{y_{1}^{\circ}, \ldots, y_{j-1}^{\circ}, y_{j+1}^{\circ}, \ldots, y_{r-1}^{\circ}\right\}$ for $1 \leq$ $j \leq r-1$ (e.g. if $[u]=\kappa_{M}^{\prime}(f)$, cf. 2.4), it yields a nulbordism once we drop the $j$ th component $u^{-1}\left(a_{j}[0, \infty)\right)$ from the extended link $u^{-1}\left(a_{0}(\mathbb{R})\right) \amalg \ldots$ constructed above; equivalently, $v$ becomes trivial when we collaps $S_{j}^{m-1}$ to a point. Hence in the Hilton decomposition (cf. [Hi]) of $v$ only those Whitehead products play a nontrivial role which involve each sphere $S_{j}^{m-1}, j=1, \ldots, r-1$, (at least) once; for dimension reasons they can therefore not involve $S_{0}^{m-1}$. Thus the extended homotopy class $v$ originates in the sub-wedge $\mathrm{V}^{r-1} S_{j}^{m-1}$; consequently, its two images $c_{a}[u]$ and $c_{\left(a_{1}^{\prime}, a_{2}, \ldots, a_{r-1}\right)}[u]$ coincide. 
The same argument applies to $\widetilde{M}$. Since the homomorphism $\widetilde{c}_{a}^{g}$ factors through $\pi_{|p|}^{\prime}\left(\widetilde{M}-\left\{g_{1}\left(\widetilde{y}_{1}^{\circ}\right), \ldots, g_{r-1}\left(\widetilde{y}_{r-1}^{\circ}\right)\right\}\right)$, it is independent of the arcs starting in the missing points $g_{j}\left(\widetilde{y}_{j}^{\circ}\right)$.

The previous result makes the transition from $M$ to $\widetilde{M}$ more transparent. Consider the lifting

$$
\widetilde{f}=\widetilde{f}_{1} \amalg \cdots \amalg \widetilde{f}_{r}: \amalg S^{p_{j}} \longrightarrow \widetilde{M}
$$

of our base point preserving $\kappa_{M}$-Brunnian link map $f$ such that $f_{j}\left(y_{j}^{\circ}\right)=\widetilde{y}_{j}^{\circ}$ for $j=1, \ldots, r$. Then for every system $g=\left(g_{1}, \ldots, g_{r-1}\right) \in G^{r-1}$ of covering transformations the lifting

$$
\widetilde{f^{g}}=g_{1} \circ \tilde{f}_{1} \amalg \cdots \amalg g_{r-1} \circ \tilde{f}_{r-1} \amalg \widetilde{f}_{r}: \amalg S^{p_{j}} \longrightarrow \widetilde{M}
$$

is $\kappa_{\widetilde{M}}$-Brunnian (and base point preserving for the obvious choice of base point $g \circ \widetilde{y}^{\circ}$ in $\widetilde{C}_{r}(\widetilde{M})$; but as we will see in $\S 3$, in our dimension range $\mu$-invariants are base point free link homotopy invariants in $\widetilde{M})$.

Corollary 2.16. If $|p| \leq r(m-2)$ then

$$
\widetilde{\mu}_{M, g}(f)=\mu_{\tilde{M}}\left(\tilde{f}^{g}\right) \quad \text { for all } g \in G^{r-1} .
$$

Proof. The constructions of the two invariants are compatible at every step. In particular, $\widetilde{c}_{a}^{g}\left(\widetilde{\kappa}_{M}^{\prime}(f)\right)$ and $c_{\tilde{a}}\left(\kappa_{\tilde{M}}^{\prime}\left(\tilde{f}^{g}\right)\right)$ are well defined (without depending on any choice of arcs) and coincide.

Finally we discuss a situation where our $\mu$-invariants can be described in terms of "linking coefficients" (and where they, therefore, turn out to be again independent of the choice of arcs involved in their construction).

Definition 2.17. $f_{1} \amalg \cdots \amalg f_{r-1}$ is said to admit a strong link nulhomotopy if it can be extended to a link map

$$
F_{1} \amalg \cdots \amalg F_{r-1}: \coprod_{j=1}^{r-1} B^{p_{j}+1} \longrightarrow M-\left\{y_{r}^{\circ}\right\}
$$

(i.e. the balls have disjoint images already in $M$, not only in $M \times I$ ).

If such a strong link homotopy exists, its intersection with $f_{r}$ yields a (nonspherical) framed link map

$$
b=\coprod_{j=1}^{r-1} b_{j}: \coprod_{j=1}^{r-1} N_{j}^{p_{j}+p_{r}+1-m} \longrightarrow S^{p_{r}}-\{*\}=\mathbb{R}^{p_{r}}
$$

as follows. After suitable approximations the maps

$$
F_{j} \times f_{r}: B^{p_{j}+1} \times S^{p_{r}} \longrightarrow M \times M
$$

are smooth and transverse to the diagonal $\Delta \subset M \times M$; the resulting manifolds $N_{j}=\left(F_{j} \times f_{r}\right)^{-1}(\Delta)$ are closed, framed (use a contraction of $B^{p_{j}+1}$ to the boundary point $* \in S^{p_{j}}$, as well as a local orientation of $M$ at $\left.f_{j}(*)\right)$, and equipped with the desired (projection) maps $b_{j}$ to $\mathbb{R}^{p_{r}}, j=1, \ldots, r-1$. Clearly, the (singular) framed link bordism class $[b]$ of $b$ (and hence also its values under the Hopf homomorphisms $h_{\gamma}$, cf. [K 5], § 3) depend only on the (pointed) homotopy class of $f_{r}$ in $M-\bigcup_{j=1}^{r-1} f_{j}\left(S^{p_{j}}\right)$. 
Example 2.19. If all $F_{j}$ are smooth embeddings and if $f_{r}$ maps into a wedge of meridians of the $f_{j}$, then $[b]$ is just the singular framed link bordism class corresponding to the linking coefficient $\left[f_{r}\right] \in \pi_{*}\left(\bigvee^{r-1} S^{m-p_{j}-1}\right)$.

Proposition 2.20. Let $f=\coprod_{j=1}^{r} f_{j}$ be a base point preserving $\kappa_{M}$-Brunnian link map such that $f_{1} \amalg \cdots \amalg f_{r-1}$ admits a strong link nulhomotopy. Assume $1 \leq p_{r} \leq m-2$. Then

$$
\pm \mu(f)=h([b])
$$

is the value of the total Hopf homomorphism $h=\oplus h_{\gamma}$ on the link map $b$ into $\mathbb{R}^{p_{r}}$ defined in 2.18.

Proof. Due to our dimension condition we may assume that $f_{r}$ avoids the arcs $a_{1}, \ldots, a_{r-1}$ used in the construction of $\mu(f)$.

Define maps

$$
\ell_{j}: S^{p_{j}} \times[0, \infty) \longrightarrow M-\left\{y_{r}^{\circ}\right\} \quad, \quad j=1, \ldots, r-1,
$$

by

$$
\ell_{j}(x, t)= \begin{cases}F_{j}((1-t) x+t *) & \text { if } t \in I=[0,1], \\ a_{j}(t-1) & \text { if } t \in[1, \infty) .\end{cases}
$$

This gives, in particular, a homotopy

$$
S^{(p)} \times I \longrightarrow \widetilde{C}_{r-1}(M),\left(x_{1}, \ldots, x_{r}, t\right) \longrightarrow\left(\ell_{1}\left(x_{1}, t\right), \ldots, \ell_{r-1}\left(x_{r-1}, t\right)\right),
$$

which can be lifted to $\widetilde{C}_{r}(M)$ via an additional component map

$$
\ell_{r}: S^{(p)} \times I \longrightarrow M
$$

in such a way that $\ell_{r}\left(x_{1}, \ldots, x_{r}, 0\right)=f_{r}\left(x_{r}\right)$ and $\ell_{r}\left(x_{1}, \ldots, x_{r-1}, *_{r}, t\right)=y_{r}^{\circ}$ for all $\left(x_{1}, \ldots, x_{r}, t\right) \in S^{(p)} \times I$. In other words, we obtain a homotopy from the product map $\hat{f}$ of $f$ (cf. 1.4) to a map $w$ (into the fiber $M-\left\{y_{1}^{\circ}, \ldots, y_{r-1}^{\circ}\right\}$ ) which is constant on $X=S^{p_{1}} \times \cdots \times S^{p_{r-1}} \times\left\{*_{r}\right\}$. Thus, by intersecting $w$ with the arcs $a_{j}$, we obtain a framed link $\bar{w}$ in $S^{(p)}-X=S^{p_{1}} \times \cdots \times S^{p_{r-1}} \times \mathbb{R}^{p_{r}}$. We have $\mu(f)= \pm h(\bar{w})$ (compare diagram 5 and proposition 2.2 in [K 5]).

But now consider the intersections (for $j=1, \ldots, r-1$ )

$$
\left(\ell_{r} \pitchfork \ell_{j}\right)^{+}:=\left\{(x, t, \tau) \in S^{(p)} \times I \times[0, \infty) \mid \ell_{r}(x, t)=\ell_{j}(x, \tau), t<\tau\right\} .
$$

Together with obvious maps (projections) into $S^{(p)}-X$ they yield a framed singular bordism between (at $t=1$ ) the link $\bar{w}$ defined above and (at $t=0$ ) a link map of the form

$$
\coprod_{j=1}^{r-1} \operatorname{id}_{S^{p_{1}} \times \cdots \times \widehat{S^{p_{j}}} \times \ldots S^{p_{r-1}}} \times\left(b_{j}^{\prime}, b_{j}\right): N_{j} \longrightarrow S^{p_{j}} \times \mathbb{R}^{p_{r}}
$$

where $b_{j}$ is as in $2.18 ; \quad b_{j}^{\prime}$ can be made constant after an additional link bordism. We can therefore conclude (compare [K 5], 6.1) that

$$
\pm \mu(f)=h(\bar{w})= \pm h(b)
$$




\section{$\S 3$. The base point free version of the $\widetilde{\mu}$-invariant}

In this section we study the effect of (link) homotopies which do not preserve base points.

There is a canonical isomorphism

$$
\pi_{1}\left(\widetilde{C}_{r}(M), y^{0}\right) \cong \pi_{1}\left(M^{r}, y^{0}\right) \cong \bigoplus_{j=1}^{r} \pi_{1}\left(M, y_{j}^{0}\right) \cong G^{r} .
$$

Moreover the base point free homotopy set $\left[S^{(p)}, \widetilde{C}_{r}(M)\right]$ is just the orbit set of the following standard action of this group on $\left[\left(S^{(p)}, *\right),\left(\widetilde{C}_{r}(M), y^{0}\right)\right]:$ given $\bar{g}=$ $\left(\bar{g}_{1}, \ldots, \bar{g}_{r}\right) \in G^{r}$ and $v:\left(S^{(p)}, *\right) \longrightarrow\left(\widetilde{C}_{r}(M), y^{0}\right)$, deform $v$ until it is constant on an $\varepsilon$-ball $B(*, \varepsilon)$ around the base point $*$ and then replace $v \mid B(*, \varepsilon)$ by the map $x \longrightarrow c(\|x\| / \varepsilon)$; here $\left[c=\left(c_{1}, \ldots, c_{r}\right)\right] \in \pi_{1}\left(\widetilde{C}_{r}(M), y^{0}\right)$ corresponds to $\bar{g}$.

Actually, we may assume that the paths $c_{j}: I \longrightarrow M$ are disjoint and smooth, embedding $(0,1)$, with liftings $\widetilde{c}_{j}$ in $\widetilde{M}$ which join $\widetilde{c}_{j}(0)=\widetilde{y}_{j}^{0}$ to $\widetilde{c}_{j}(1)=$ $\bar{g}_{j}\left(\widetilde{y}_{j}^{0}\right), j=1, \ldots, r$. Pick an isotopy $L: M \times I \longrightarrow M$ (from the identity map $\operatorname{id}_{M}$ to some base point preserving diffeomorphism $\ell_{\bar{g}}$ ) which pushes $y_{j}^{\circ}$ along $c_{i}$, i.e. $L\left(y_{j}^{\circ}, t\right)=c_{j}(t)$, for $1 \leq j \leq r$. This lifts to an isotopy $\widetilde{L}$ from $\operatorname{id}_{\widetilde{M}}$ to some self-diffeomorphism $\widetilde{\ell}_{\bar{g}}$ of $\widetilde{M}$ satisfying $\tilde{\ell}_{\bar{g}}\left(\gamma \widetilde{y}_{j}^{0}\right)=\gamma \bar{g}_{j}\left(\widetilde{y}_{j}^{0}\right)$ for all $\gamma \in G$ and $j \leq r$.

Clearly the induced selfmaps $\ell_{\bar{g} *}$ of the homotopy sets in diagram 2.5 commute with the arrows there. Moreover, $\ell_{\bar{g} *}$ yields the action of $\bar{g}^{-1}$ on the set $\left[\left(S^{(p)}, *\right),\left(\widetilde{C}_{r}(M), y^{0}\right)\right]$.

On the other side consider the effect of $\ell_{\bar{g} *}$ on (the image $[u]=p_{*}([\widetilde{u}])$ of) an element $[\widetilde{u}]$ in the top right hand group in diagram 2.6. $\widetilde{\ell}_{\bar{g}} \circ \bar{g}_{r}^{-1} \circ \widetilde{u}$ is the lifting of $\ell_{\bar{g}} \circ u$ which maps $*$ to $\widetilde{y}_{r}^{\circ}$ and which therefore represents the homotopy class $p_{*}^{-1}\left(\ell_{\bar{g} *}([u])\right)$. Its value under $\widetilde{c}_{a}^{g}, g \in G^{r-1}$, is given by the intersections of $\widetilde{\ell}_{\bar{g}} \circ \bar{g}_{r}^{-1} \circ \widetilde{u}$ with the arcs $g_{j} \circ \widetilde{a}_{j}$ (which start at $g_{j}\left(\widetilde{y}_{j}^{\circ}\right), j=1, \ldots, r-1$ ) or also by the intersections of $\widetilde{u}$ with the arcs $\bar{g}_{r} \circ \widetilde{\ell}_{\bar{g}}^{-1} \circ g_{j} \circ \widetilde{a}_{j} \quad\left(\right.$ which start at $\bar{g}_{r} g_{j} \bar{g}_{j}^{-1}\left(\widetilde{y}_{j}^{\circ}\right)$ ). Hence, for a suitable system $\bar{a}$ of arcs we have in 2.6

$$
h \circ \widetilde{c}_{a}^{g}\left(p_{*}^{-1}\left(\ell_{\bar{g} *}([u])\right)\right)=\operatorname{sign}\left(\bar{g}_{r}\right)^{r-1} \cdot h \circ \widetilde{c}_{\bar{a}}^{g^{\prime}}\left(p_{*}^{-1}([u])\right)
$$

where $g^{\prime}=\bar{g}^{-1} * g$ and we define

$$
\bar{g} * g:=\left(\bar{g}_{r}^{-1} g_{1} \bar{g}_{1}, \ldots, \bar{g}_{r}^{-1} g_{r-1} \bar{g}_{r-1}\right) \in G^{r-1}
$$

whenever $\bar{g}=\left(\bar{g}_{1}, \ldots, \bar{g}_{r}\right) \in G^{r}$ and $g=\left(g_{1}, \ldots, g_{r-1}\right) \in G^{r-1}$.

If $|p| \leq r(m-2)$, then by proposition $2.15 \quad \widetilde{c}^{g^{\prime}}:=\widetilde{c}_{\bar{a}}^{g^{\prime}}=\widetilde{c}_{a}^{g^{\prime}}$ is independent of any choice of arcs and depends only on the superscript $g^{\prime} \in G^{r-1}$; moreover, the action of $\bar{g} \in G^{r}$ on any of the homotopy sets in 2.5 or in the top line of 2.6 corresponds just to the transformation $g \longrightarrow \bar{g} * g$ of the superscripts $g \in G^{r-1}$ in $\oplus h \circ \widetilde{c}^{g}$, together with multiplication by -1 if $\bar{g}_{r}$ reverses the orientation of $\widetilde{M}$ and $r$ is even. We conclude 
Proposition and Definition 3.3. Assume $|p| \leq r(m-2)$. Given any link map $f$ (as in 1.3) which is $\kappa-$ Brunnian, pick a base point preserving representative $f^{b}$ of its link homotopy class. Then the orbit

$$
\left[\widetilde{\mu}\left(f^{b}\right)=\left\{\widetilde{\mu}_{g}\left(f^{b}\right)\right\}\right] \in\left(\bigoplus_{g \in G^{r-1}}\left(\pi_{*}^{S}\right)^{(r-2) !}\right) / G^{r}
$$

(w.r. to the action of $G^{r}$ described above) depends only on the base point free link homotopy class of $f$ and is called the base point free $\widetilde{\mu}$-invariant of $f$.

Corollary 3.4. If in addition $M$ is orientable, then $\mu(f)$ (cf. 2.9) depends only on the base point free link homotopy class of $f$.

Indeed, in this case the action of $G^{r}$ leaves $\mu(f)= \pm \Sigma \widetilde{\mu}_{g}(f)$ invariant (cf. 2.12).

\section{$\S 4$. Compatibility with linking coefficients}

Throughout this section assume that $1 \leq p_{1}, \ldots, p_{r-1} \leq m-2$ while $p_{r} \geq 1$ is arbitrary unless specified otherwise.

Pick a closed subset $A$ of $M$ as in condition 2.1 and identify it with $\mathbb{R}^{m-1} \times[0, \infty)$; after appropriate isotopies we may assume that

$$
y_{j}^{\circ}=(j, 0, \ldots, 0 ; 2) \in \stackrel{\circ}{A}, \quad j=1, \ldots, r-1,
$$

and $y_{r}^{\circ} \in \partial A$. Using a wedge of suitable small $m$-balls $B_{j} \subset A$ such that $y_{j}^{\circ} \in \stackrel{\circ}{B}_{j}$, but $y_{j}^{\circ} \notin B_{k}$ for $j \neq k, 1 \leq j, k \leq r-1$, as well as of their boundary spheres $S_{j}^{m-1}=\partial B_{j}$, we obtain homotopy equivalences

$$
M \vee \bigvee_{j=1}^{r-1} S_{j}^{m-1} \sim(M-\stackrel{\circ}{A}) \vee \bigvee_{j=1}^{r-1} S_{j}^{m-1} \sim M-\left\{y_{1}^{\circ}, \ldots, y_{r-1}^{\circ}\right\}
$$

which preserve the common basepoint $y_{r}^{0}$ and consist of inclusions and deformation retractions. Thus, in diagram 2.6 we may consider $\kappa^{\prime}(f)$ as a homotopy class in the wedge of $M-\stackrel{\circ}{A}$ (or of $M$, resp.) with $\bigvee S_{j}^{m-1}$, and (for the choice of arcs $a_{j}(t):=(j, 0, \ldots, 0 ; t+2)$ and obvious local orientations) we may interpret $c_{a}$ as a localization homomorphism since it is induced by the map which collapses the complement of the open cell $\stackrel{\circ}{A}$ in $M$ (or all of $M$, resp.) to a point.

Next, given $p_{j} \leq m-2$, let $\ell_{j}$ be a base point preserving standard embedding of $S^{p_{j}}$ into $\{j\} \times \mathbb{R}^{m-2} \times(0,2] \subset \stackrel{\circ}{A}$ (with image $e_{j}$, cf. 4.3 ), $j=1, \ldots, r-1$. In analogy to 4.2 , a suitable choice of corresponding meridians leads to a map

$$
e: M \vee \bigvee_{j=1}^{r-1} S_{j}^{q_{j}} \sim(M-\stackrel{\circ}{A}) \vee \bigvee_{j=1}^{r-1} S_{j}^{q_{j}} \longleftrightarrow M-\bigvee_{j=1}^{r-1} \ell_{j}\left(S^{p_{j}}\right)
$$

where $q_{j}:=m-p_{j}-1$. Thus given any map $\ell_{r}: S^{p_{r}} \longrightarrow M \vee \bigvee S_{j}^{q_{j}}$, we obtain the link map

$$
e_{*}\left(\ell_{r}\right):=\ell_{1} \amalg \cdots \amalg \ell_{r-1} \amalg e_{\circ} \ell_{r} \quad .
$$


Figure 4.3. The construction of $e_{*}$

Example 4.6 (Homotopy Brunnian links). Assume that $M=M^{\prime} \times \mathbb{R}$ for some smooth $(m-1)$-manifold $M^{\prime}$ and that $1 \leq p_{1}, \ldots, p_{r} \leq m-3$. After pushing two link maps $f_{+}, f_{-}$suitably into opposite halfspaces $M_{ \pm}=M^{\prime} \times[0, \pm \infty)$ we can form a connected sum $f_{+}+f_{-}$with the property that $f_{+}+f_{-} \sim f_{+}$if $f_{-}$ is homotopy trivial (cf. 1.7).

Now let $f$ be a link (i.e. embedding) which is homotopy Brunnian (cf. 1.7). Then after a suitable isotopy the components $f_{1}, \ldots, f_{r-1}$ intersect $M_{-}$in small disjoint standard halfspheres. In their complement $f_{r}$ can be deformed into a wedge of $M^{\prime} \times\{-1\}$ (or, better yet, of $M^{\prime} \times\{0\}-$ open ball) with meridians $S_{j}^{q_{j}} \subset M_{-}, 1 \leq j \leq r-1$ (use Thom isomorphisms and apply the Whitehead theorem to the universal cover of the link complement). Now perform a (partial) link nulhomotopy in $M_{+}$until $f=e_{*}\left(f_{r}\right)$. In order to insure that $\left[f_{r}\right]$ is a reduced homotopy class add correction terms of the form $e_{*}\left(-\left[c \circ f_{r}\right]\right)$ where $c$ collapses a (decreasing) number of meridians. Since $f$ is homotopy Brunnian this iteration procedure does not change the link homotopy class of $f$ which therefore lies in the image of the reduced homotopy group $\pi_{p_{r}}^{\prime}\left(M \vee \bigvee S_{j}^{q_{j}}\right)$ under $e_{*}$.

(In this example the choice of $A$ is based on a chart of $M^{\prime}$ and on the decomposition $\left.M=M^{\prime} \times \mathbb{R}\right)$.

In general for any integer $p_{r} \geq 0$ the diagram

$$
\begin{aligned}
& \pi_{p_{r}}^{\prime}\left(M \vee \bigvee_{j=1}^{r-1} S_{j}^{q_{j}}, y_{r}^{0}\right) \stackrel{e_{*}}{\longrightarrow}\left\{\begin{array}{c}
\text { b. point pres. link } \\
\text { homotopy classes } \\
\text { of } \kappa_{M}^{-} \varepsilon_{\text {Bunnian }} \\
f: \amalg_{j=1}^{r} S_{S^{p_{j}} \rightarrow M}
\end{array}\right\} \stackrel{\kappa_{M}^{\prime}}{\longrightarrow} \pi_{|p|}^{\prime}\left(M \vee \bigvee_{j=1}^{r-1} S_{j}^{m-1}, y_{r}^{0}\right) \\
& \text { quot* }\rceil \\
& {\left[\left(S^{(p)}, *\right),\left(M \vee \bigvee_{j=1}^{r-1} S_{j}^{m-1}, y_{r}^{0}\right)\right]}
\end{aligned}
$$

relates the homotopy groups (which are reduced w.r. to the spheres $S_{j}^{q_{j}}$ and $S_{j}^{m-1}$, compare 2.4 and the formula following 2.6) of the spaces in 4.4 ("input") and 4.2 ("output"). Here the map $\varepsilon$ can be interpreted as some kind of (iterated) nonspherical Nezhinskij suspension (compare e.g. $\S 7$ of [K 5]). It is constructed 
as follows. Given points $z_{j} \in S_{j}^{q_{j}}-\{*\}, j=1, \ldots, r-1$, any map $\ell_{r}: S^{p_{r}} \longrightarrow$ $M \vee \bigvee S_{j}^{q_{j}}$ can be deformed until there are disjoint compact tubular neighborhoods $T_{j}$ in $S^{p_{r}}$ of each $\left(m-1-p_{j}\right)$-codimensional submanifold $N_{j}=\ell_{r}^{-1}\left(z_{j}\right)$ and $\ell_{r}$ restricts to

$$
\ell_{r} \mid:\left(T_{0}:=S^{p_{r}}-\bigcup_{j=1}^{r-1} \stackrel{\circ}{T}_{j}, \partial T_{0}\right) \longrightarrow\left(M, y_{r}^{\circ}\right)
$$

In $S^{(p)}=S^{p_{1}} \times \cdots \times S^{p_{r}}$ this gives rise to the framed $(m-1)$-codimensional submanifolds

$$
\widehat{N}_{j}=S^{p_{1}} \times \cdots \times S^{p_{j-1}} \times\left\{-*_{j}\right\} \times S^{p_{j+1}} \times \cdots \times S^{p_{r-1}} \times N_{j}
$$

and to the map $\ell_{r}$ o projection on $S^{p_{1}} \times \cdots \times S^{p_{r-1}} \times T_{0}$. These data fit together and define $\varepsilon\left(\left[\ell_{r}\right]\right)$ via the Pontryagin-Thom construction.

Recall that by a result of D. Puppe the obvious map quot* in 4.7 is known to be injective (see $[\mathrm{P}]$, Satz 12, 13, and 20).

Proposition 4.9. For all $0 \leq p_{1}, \ldots, p_{r-1} \leq m-2, \quad p_{r} \in \mathbb{Z}$ and $M$ as in 2.1 the composite $\kappa_{M}^{\prime} \circ e_{*}$ in 4.7 is characterized by the identity

$$
\text { quot* }^{*} \circ \kappa_{M}^{\prime} \circ e_{*}=\operatorname{invol}_{*} \circ \varepsilon
$$

where invol $=\mathrm{id}_{M} \vee \bigvee b_{j}$ is a suitable involution of $M \vee \bigvee S_{j}^{m-1}$ such that the selfmap $b_{j}$ of $S_{j}^{m-1}$ has degree $\pm 1, \quad j=1, \ldots, r-1$.

Proof. Choose isotopies $d_{y_{j}, t}: M \longrightarrow M$ which

(i) depend smoothly on $y_{j} \in S^{p_{j}}-\{*\}$ and $t \in I$;

(ii) deform $\ell_{j}\left(y_{j}\right)$ to $y_{j}^{\circ}$ along a straight arc in $A$; and

(iii) leave all points outside of a small neighbourhood of this arc fixed.

Then, given a generic map $\ell_{r}: S^{p_{r}} \longrightarrow M \vee \bigvee S_{j}^{q_{j}}$, the homotopy $S^{(p)} \times I \longrightarrow$ $\widetilde{C}_{r}(M)$,

$$
\left(y_{1}, \ldots, y_{r} ; t\right) \longrightarrow d_{y_{1}, t} \circ \ldots \circ d_{y_{r-1}, t}\left(\ell_{1}\left(y_{1}\right), \ldots, \ell_{r-1}\left(y_{r-1}\right), e \circ \ell_{r}\left(y_{r}\right)\right)
$$

deforms the product map $k_{0}$ of $e_{*}\left(\ell_{r}\right)$ to a map $k_{1}$ into $M-\left\{y_{1}^{\circ}, \ldots, y_{r-1}^{\circ}\right\}$ which, when composed with the homotopy equivalence in 4.2 , represents $\varepsilon\left(\left[\ell_{r}\right]\right.$ ) (possibly up to a change of the framings of the $(m-1)$-codimensional submanifolds listed in 4.8). The same procedure allows to deform a nulhomotopy of $k_{0} \mid X_{j}$ (cf. 1.7) to a nulhomotopy of $k_{1} \mid X_{j}, j=1, \ldots, r$. Therefore, if $\left[\ell_{r}\right]$ is reduced, then $\left[k_{1}\right]=$ quot $^{*}\left(\left[k^{\prime}\right]\right)$ for some $\left[k^{\prime}\right] \in \pi_{|p|}\left(M-\left\{y_{1}^{\circ}, \ldots, y_{r-1}^{\circ}\right\}\right)$; but for injectivity reasons, $\left[k^{\prime}\right]$ must equal $\kappa_{M}^{\prime}\left(e_{*}\left(\ell_{r}\right)\right)$ (compare 2.5).

Next let $\iota_{j}$ (and $\bar{\iota}_{j}$, resp.) denote the homotopy class of the inclusion of $S_{j}^{q_{j}}$ into $M \bigvee \bigvee S_{j}^{q_{j}}$ (and of $S_{j}^{m-1}$ into $M \bigvee \bigvee S_{j}^{m-1}$, resp.), $j=1, \ldots, r-1$. Moreover, for every $g \in \pi_{1}\left(M, y_{r}^{0}\right)=G$, let $g \iota_{j}$ and $g \bar{\iota}_{j}$ be defined by the canonical action of $G$. 
Theorem 4.10. Assume that $1 \leq p, \ldots, p_{r-1} \leq m-2$ and $p_{r} \geq 1$.

Then the composite map $\kappa_{M}^{\prime} \circ e_{*}$ in 4.7 is a group homomorphism.

Furthermore, it is compatible with Whitehead products. More precisely, let $w \in$ $\pi_{d}^{\prime}\left(M \vee \vee S_{j}^{q_{j}}\right)$ be any Whitehead product which involves at least one factor of the form $g_{j} \iota_{j}, g_{j} \in G$, for each $j=1, \ldots, r-1$ and possibly further factors in $\pi_{*}(M), * \geq 2$; let $\bar{w} \in \pi_{*}\left(M \vee \bigvee S_{j}^{m-1}\right)$ denote the corresponding Whitehead product with $\iota_{j}$ replaced by $\bar{\iota}_{j}, j=1, \ldots, r-1$.

If each $\iota_{j}$ occurs precisely once in $w$ then the diagram

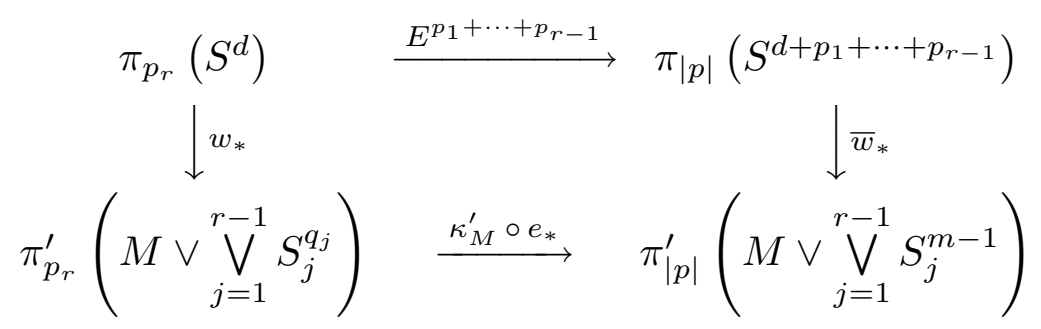

commutes up to a \pm sign; here $E$ denotes suspension.

If for some $1 \leq j_{0} \leq r-1$ more than one factor of the form $g \cdot \iota_{j_{0}}, g \in G$, occurs in $w$, then the homomorphism $\kappa_{M}^{\prime} \circ e_{*} \circ w_{*}$ is trivial.

Thus $\kappa_{M}^{\prime} \circ e_{*} \circ w_{*}= \pm \bar{w}_{*} \circ E^{|p|-p_{r}}$ whenever the dimensions of $\bar{w}$ and $w$ differ by $|p|-p_{r}$; otherwise $\kappa_{M}^{\prime} \circ e_{*} \circ w_{*}=0$.

Proof. We may assume in 4.5 that each $\ell_{j}\left(S^{p_{j}}\right) \subset A$ is symmetric w.r. to a hyperplane $H$ which contains all the base points and chosen meridians. Given $\ell_{+}, \ell_{-} \in \pi_{p_{r}}^{\prime}\left(M \vee \bigvee S_{j}^{q_{j}}\right)$, consider $e_{*}\left(\ell_{+}+\ell_{-}\right)$. In the summand $e_{\circ} \ell_{+}$(and $\left.e \circ \ell_{-}\right)$ move the meridian $S_{j}^{q_{j}}$ to the positive (and negative, resp.) side of $H$; then pinch the equator $\ell_{j}\left(S^{p_{j}}\right) \cap H$ to a point. Thus $\kappa_{M}^{b} \circ e_{*}\left(\ell_{+}+\ell_{-}\right)$factors through $\prod\left(S_{+}^{p_{j}} \vee S_{-}^{p_{j}}\right)$ and is nulhomotopic on each of the subtori except on $\prod S_{+}^{p_{j}}$ and $\prod S_{-}^{p_{j}}$. A cell-by-cell argument shows that

$$
\kappa_{M}^{\prime} \circ e_{*}\left(\ell_{+}+\ell_{-}\right)=\kappa_{M}^{\prime} \circ e_{*}\left(\ell_{+}\right)+\kappa_{M}^{\prime} \circ e_{*}\left(\ell_{-}\right)
$$

Next, given $1 \leq j \leq r-1$, consider a subproduct of $w$ of the form

$$
v= \pm\left[\iota_{j}, u\right], \quad u \in \pi_{n}\left(M \vee \bigvee_{i \neq j} S_{i}^{q_{i}}\right)
$$

Geometrically $v$ can be described by

(i) a framed standard sphere $N_{j}=S^{n-1} \subset \mathbb{R}^{q_{j}+n-1}$, and

(ii) the composite map

$$
\partial B^{q_{j}} \times \mathbb{R}^{n} \stackrel{\text { projection }}{\longrightarrow} \mathbb{R}^{n} \subset S^{n} \stackrel{u}{\longrightarrow} M \vee \bigvee_{i \neq j} S_{i}^{q_{i}}
$$

defined on a small tubular neighbourhood of the meridian $\partial B^{q_{j}}$, where $B^{q_{j}}$ denotes a $q_{j}$-ball normal to $N_{j}$ and with center at some point $z \in N_{j}$.

Now apply the $\varepsilon$-construction (w.r. to products with $S^{p_{j}}$, cf. 4.8 ) to $v$ and compare the result to the analogous geometric description of $\bar{v}= \pm\left[\bar{\iota}_{j}, u\right]$ in 
$\mathbb{R}^{(m-1)+n-1} \subset \mathbb{R}^{q_{j}+n-1} \times S^{p_{j}}$. Remove the interior of a small ball $B^{m-1}$ around $\left(z,-*_{j}\right)$ from $B^{q_{j}} \times S^{p_{j}}$ to obtain a framed link bordism (in $\mathbb{R}^{q_{j}+n-1} \times S^{p_{j}} \times I$ ) which allows us to replace $\partial B^{q_{j}} \times S^{p_{j}}$ by $\partial B^{m-1}$ in (ii) above while leaving $N_{j}$ unchanged.

If $v= \pm g \iota_{j}, g \in G$, the $\varepsilon$-construction on $v$ is related to $\bar{v}= \pm g \bar{\iota}_{j}$ in a similar way.

In any case, whether $\pm v$ equals $\left[\iota_{j}, u\right]$ or $g \iota_{j}$, our bordism can be used fiberwise in suitable product situations (for the fiberwise geometric interpretation of iterated Whitehead products etc. compare [K 5], p. 308-309, e.g. (17)). Since the support of $\bar{v}$ lies in a (small) cell, we can apply the same argument to the product of $v$ with the next factor which occurs in $w$, provided this factor does not involve $\iota_{j}$.

To prove the commutativity claim in 4.10 we iterate this procedure w.r. to all factors in $w$ and w.r. to all $j$. We conclude that (in the notation of 4.7 and 4.9)

$$
\operatorname{quot}^{*}\left(\kappa_{M}^{\prime} \circ e_{*}(w)\right)=\operatorname{invol}_{*} \circ \varepsilon(w)=\operatorname{quot}^{*}( \pm \bar{w}) .
$$

Moreover recall that elements of $\pi_{p_{r}}\left(S^{d}\right)$ correspond - up to bordism - to open embeddings of the form $Q \times \mathbb{R}^{d} \subset \mathbb{R}^{p_{r}}$. Again we apply our construction fiberwise to every normal slice and we use the fact that quot* is injective to obtain finally the desired commutativity.

In this proof we need only one Whitehead factor $g_{j} \iota_{j}$ for each $1 \leq j \leq r-1$ in order to substitute $S^{p_{j}}$ by a small cell $c_{j}$ around $-*_{j}$ and thus to describe (quot $\left.^{*}\right)^{-1} \circ \varepsilon$ (compare 4.7). Now suppose that some $\iota_{j}$ occurs at least twice in $w$, i.e. $w$ contains a subproduct of the form $\left[v_{1}, v_{2}\right]$ where both $v_{1}$ and $v_{2}$ involve $\iota_{j}$ precisely once. Then in the construction of $\varepsilon\left(\left[v_{1}, v_{2}\right]\right)$ the support of each factor $v_{i}$ may be assumed to lie in the product of a small cell $c_{j}^{(i)} \subset S^{p_{j}}$ with the remaining spheres. We may shift these cells to disjoint $S^{p_{j}}$-levels; then at each such level $\varepsilon\left[v_{1}, v_{2}\right]$ can be described by a Whitehead product with a trivial factor. We obtain a nulbordism which can again be used fiberwise to show that $\varepsilon \circ w_{*}$ and hence $\kappa_{M}^{\prime} \circ e_{*} \circ w_{*}$ must be trivial (compare proposition 4.9).

Now suppose $|p| \leq r(m-2)$ (so that we have e.g. 2.15 at our disposal). If in the discussion above $w$ is just a Whitehead product of $r-1$ factors of the form $g_{1} \iota_{1}, \ldots, g_{r-1} \iota_{r-1}$, all $g_{j} \in G$, then $E^{p_{1}+\cdots+p_{r-1}}$ is the stable suspension on the domain of $w_{*}$; more importantly, $\kappa_{M}^{\prime} \circ e_{*} \circ w_{*}$ is detected by $\widetilde{h}_{\circ}\left(\oplus \widetilde{c}_{a}^{g}\right) \circ p_{*}^{-1}$ (cf. 2.6 and the remarks following 4.2) and hence $\widetilde{\mu}_{M}$ is just as strong as $\kappa_{M}^{\prime}$ on the image of $e_{*} \circ w_{*}$. However, if $w$ involves all $\iota_{j}$ and an additional factor $g \iota_{j}$ or $b \in \pi_{n}(M), n \geq 2$, then $\widetilde{\mu}_{M} \circ e_{*} \circ w_{*} \equiv 0$.

As a consequence we see that in general $\kappa_{M}^{\prime}$ can be strictly stronger than $\widetilde{\mu}_{M}$.

Example 4.11. Suppose that for some continuous map $g: M \longrightarrow S^{n}$ and some $b \in \pi_{n}(M), 2 \leq n \leq m-2$, the homotopy class $g_{*}(b) \in \pi_{n}\left(S^{n}\right)$ is nontrivial. Consider the Whitehead product

$$
w=\left[\ell_{r}\right]=\left[\iota_{1},\left[\iota_{2}, \ldots,\left[\iota_{r-1}, b\right] \ldots\right]\right] \in \pi_{d}^{\prime}\left(M \vee \bigvee_{j=1}^{r-1} S_{j}^{q_{j}}\right) .
$$

Then the $\kappa_{M}-$ Brunnian link map

$$
e_{*}\left(\ell_{r}\right): \coprod_{j=1}^{r-1} S_{j}^{p_{j}} \amalg S^{d} \longrightarrow M
$$


(cf. 4.5) has trivial $\widetilde{\mu}$-invariant but $\kappa_{M}^{\prime}\left(e_{*}\left(\ell_{r}\right)\right)= \pm \bar{w} \neq 0$. Indeed, when suitably mapped to the wedge $S^{n} \vee \bigvee S_{j}^{m-1}, \bar{w}$ is nontrivial there (see e.g. [K 5], 3.1).

According to the last claim in theorem 4.10 the product map $\widehat{f}$ (cf. 1.4) often allows a (standard) nulhomotopy. When can this be induced by a link nulhomotopy?

Conjecture 4.12. Let $w \in \pi_{d}^{\prime}\left(M \vee \bigvee S^{q_{j}}\right)$ be a Whitehead product as in 4.10 containing two factors of the form $g \iota_{j_{0}}, g^{\prime} \iota_{j_{0}}$ for some $1 \leq j_{0} \leq r-1$. Then for every $u \in \pi_{p_{r}}\left(S^{d}\right)$ the link map $e_{*}\left(w_{*}(u)\right)$ is trivial.

Here is a partial result in this direction.

Proposition 4.13. Let $w \in \pi_{d}\left(M \bigvee \bigvee S^{q_{j}}\right)$ be a Whitehead product which contains a subproduct of the form $\left[g \iota_{j}, g^{\prime} \iota_{j}\right], g, g^{\prime} \in G$, for some $1 \leq j \leq r-1$. Assume $p_{1}, \ldots, p_{r} \leq m-2$.

Then for any two elements $v_{1}, v_{2} \in \pi_{p_{r}}\left(M \vee \bigvee S^{q_{j}}\right)$ such that $v_{1}-v_{2} \in$ $w_{*}\left(\pi_{p_{r}}\left(S^{d}\right)\right)$ we have

$$
e_{*}\left(v_{1}\right)=e_{*}\left(v_{2}\right) \text {. }
$$

Proof. By finger moves along suitable paths we can isotop two small portions of $\ell_{j}\left(S^{p_{j}}\right)$ (together with the meridians $g \iota_{j}$ and $\left.g^{\prime} \iota_{j}\right)$ until they face each other "perpendicularly" as in the proof of theorem 2.5 in [K 2]. Then, after a selfintersection of $f_{j}$, the Whitehead product of the two meridians becomes nulhomotopic. In other words, we may replace the subproduct $\left[g \iota_{j}, g^{\prime} \iota_{j}\right]$ of $w$ by 0 . Since $p_{r} \leq m-2$, such deformations of $f_{j}$ and $f_{r}$ do not intersect the remaining (generic) components of $e_{*}\left(v_{1}\right)$ or $e_{*}\left(v_{2}\right)$.

Example 4.14. If $r=2, p_{1} \leq m-3, p_{2} \leq m-2$ and $\widetilde{M}$ is $p_{2}$-connected, then $e_{*}$ kills all higher Hilton summands in

$$
\pi_{p_{2}}^{\prime}\left(M \vee S^{q_{1}}\right) \cong \pi_{p_{2}}^{\prime}\left(\widetilde{M \vee S^{q_{1}}}\right) \cong \pi_{p_{2}}\left(\bigvee_{g \in G} S_{g}^{q_{1}}\right) \cong \bigoplus_{g \in G} \pi_{p_{2}}\left(S_{g}^{q_{1}}\right) \oplus \ldots
$$

whereas the first order, $\pi_{p_{2}}\left(S_{g}^{q_{1}}\right)$-valued components of the linking coefficients are detected, up to stable suspension, by $\widetilde{\mu}$.

In the very special case when $M=\mathbb{R} P^{5} \times \mathbb{R}$ there are precisely 4 (or 3, resp.) base point preserving (or base point free, resp.) homotopy classes of link maps $f: S^{3} \amalg S^{4} \longrightarrow M$ which embed $S^{3}$, and they are fully determined by $\widetilde{\mu}$. Similarly, when $M=\left(S^{1}\right)^{5} \times \mathbb{R}$ there are infinitely many such homotopy classes, again distinguished by $\widetilde{\mu}$. What about general link maps where $f_{1}$ may be a (selftransverse) immersion? How many additional link homotopy classes can be detected by a "Kirk-invariant" (compare [Ki]) which counts the double points of $f_{1}$ and measures their linking behaviour with respect to $f_{2}$ ?

\section{$\S 5$. Link maps with codimensions at least 2}

Throughout this section we assume that $p_{1}, \ldots, p_{r} \leq m-2$. This implies, in particular, that $|p| \leq r(m-2)$ and proposition 2.15 applies. Moreover, a link map $f$ (as in 1.3) is $\kappa_{M}$-Brunnian if (and only if) each proper sub-link map of $f$ is $\kappa_{M}$-trivial (cf. 1.7); indeed, after a small deformation the sub-link map 
$\varphi_{j}=f_{1} \amalg \cdots \amalg f_{j-1} \amalg f_{j+1} \amalg \cdots \amalg f_{r}$ misses a path which moves $f_{j}\left(*_{j}\right)$ to a "faraway point" (lying outside of the componentwise images of a nulhomotopy of $\widehat{\varphi}_{j}$, cf. 1.4).

Here is another important consequence of our dimension assumption. Without gain or loss of information the (open) manifold $M$ can be punctured at the points of a closed discrete subset $D \subset M-\left\{y_{1}^{\circ}, \ldots, y_{r}^{\circ}\right\}$ : link maps into $M-D$ and into $M$ (considered up to link homotopy) correspond bijectively to one another via the inclusion map incl, and this correspondence is compatible with $\kappa, \mu$ - and $\widetilde{\mu}$-invariants.

Proposition 5.1. In the situation above let $f, f^{\prime}: \amalg S^{p_{j}} \longrightarrow M-D$ be two link maps. Then both in the base point preserving and in the base point free setting $f$ and $f^{\prime}$ have equal $\kappa_{M-D}$-invariants if (and only if) incl $\circ f$ and incl $\circ f^{\prime}$ have equal $\kappa_{M}$-invariants.

Proof. After an approximation we may assume that $f$ and $f^{\prime}$ are generic immersions. Let $F=\left(F_{1}, \ldots, F_{r}\right)$ be a homotopy in $\widetilde{C}_{r}(M)$ which joins their product maps $\widehat{f}$ and $\widehat{f}^{\prime}$. Then $S:=\bigcup F_{j}\left(S^{(p)} \times I\right)$ intersects $D$ only in a finite set $\left\{d_{k}\right\}$. Pick suitable disjoint arcs $a_{k}$ which start in $\left\{d_{k}\right\}$ and end at some "faraway points" $\left\{c_{k}\right\} \subset M-S$. An isotopy in small tubular neighbourhoods along these arcs yields a diffeomorphism $b$ which maps $\left\{d_{k}\right\}$ to $\left\{c_{k}\right\}$ and leaves $f$ and $f^{\prime}$ as well as all other points of $D$ unchanged. Composing $F$ with $b^{-1}$ we obtain the desired homotopy from $\widehat{f}$ to $\widehat{f}^{\prime}$ in $\widetilde{C}_{r}(M-D)$.

Corollary 5.2. A link map $f: \amalg S^{p_{j}} \longrightarrow M-D$ is $\kappa_{M-D}$-Brunnian if and only if incl $\circ f$ is $\kappa_{M}$-Brunnian; if this condition holds, then the $\widetilde{\mu}$-invariants of $f$ and incl $\circ f$ coincide both in the base point preserving and base point free setting.

Remark 5.3. Whenever $p_{1}, \ldots, p_{r} \leq m-2$, the previous discussion allows us to extend our construction of $\mu$ - and $\widetilde{\mu}$-invariants to the case when $M$ does not necessarily satisfy condition 2.1 (e.g. when $M$ is a closed manifold). Indeed, choose any nonempty closed discrete subset $D \subset M-\left\{y_{1}^{0}, \ldots, y_{r}^{0}\right\}$ (e.g. consisting of a single point) and deform a (suitable) link map $f$ in $M$ until it misses $D$ (and is $\kappa_{M-D}$-Brunnian). Both in the base point preserving and in the base point free setting this procedure yields well defined link homotopy invariants

$$
\mu_{M}(f):=\mu_{M-D}(f) \quad \text { and } \quad \widetilde{\mu}_{M}(f):=\widetilde{\mu}_{M-D}(f)
$$

which are independent of the choice of the set $D$ where $M$ is punctured.

Next we turn to the central question asked in the introduction (for $M$ satisfying condition 2.1): how much information do we loose by the simplification procedure which extracts the $\widetilde{\mu}$-invariant out of the unwieldy $\kappa$-invariant of a (base point preserving) $\kappa_{M}$-Brunnian link map $f$ in $M$ ?

On one hand $\kappa_{M}^{b}(f)$ is precisely as strong as the element

$$
\widetilde{\kappa}_{M}^{\prime}(f) \in \pi_{|p|}^{\prime}(\widetilde{C}):=\bigcap_{j=1}^{r-1} \operatorname{ker}\left({\widetilde{\operatorname{fill}_{j *}}}_{j}\right)
$$

where $\widetilde{C}$ denotes the universal covering space of

$$
C:=M \vee \bigvee_{j=1}^{r-1} S_{j}^{m-1} \sim M-\left\{y_{1}^{0}, \ldots, y_{r-1}^{0}\right\}
$$


and $\widetilde{\text { fill }}_{j}$ collapses each lifting of $S_{j}^{m-1}$ to a point (compare 2.5, 2.6, 4.2, and 2.10). On the other hand, $\widetilde{\mu}_{M}(f)$ is equal - up to the isomorphism $\widetilde{h}$ (cf. 2.6 and $2.15)$ - to the value of $\widetilde{\kappa}_{M}^{\prime}(f)$ under the total homomorphism

$$
c:=\bigoplus_{g \in G^{r-1}} \widetilde{c}^{g} \quad: \quad \pi_{|p|}^{\prime}(\widetilde{C}) \longrightarrow \bigoplus_{g \in G^{r-1}} \pi_{|p|}^{\prime}\left(\bigvee_{j=1}^{r-1} S_{j}^{m-1}\right)
$$

where for $g=\left(g_{1}, \ldots, g_{r-1}\right) \quad \widetilde{c}^{g}$ is induced by collapsing all but the $g_{j}$-lifting of $S_{j}^{m-1}, j=1, \ldots, r-1$. Therefore, our question amounts to asking how much information we lose by applying $c$. Often (though certainly not always, see e.g. 4.11 and 5.9) the answer is: none at all.

Theorem 5.6. Assume $\widetilde{M}$ is $(m-2)$-connected and $p_{1}, \ldots, p_{r} \leq m-2$ and let $f: \amalg S^{p_{j}} \longrightarrow M$ be a $\kappa_{M}$-Brunnian link map. Then both in the base point preserving and in the base point free setting the $\kappa_{M}$-invariant of $f\left(\right.$ i.e. $\kappa_{M}^{b}(f)$ or $\kappa_{M}(f)$, resp.) contains precisely as much information as the $\widetilde{\mu}_{M}$-invariant ( as defined in 2.11 or 3.3 , resp.).

Proof. Choose a triangulation of $M$ (cf. [Mu], 10.6) and let $D$ be the set consisting of the barycenters of all $m$-simplices. It suffices to prove the injectivity of the total collapsing homomorphism $c$ as in 5.5, but with $M$ replaced by $\dot{M}:=M-D$.

Now $\widetilde{\dot{M}}$ is $(m-2)$-connected and - since it contains the $(m-1)$-skeleton as a deformation retract - has a free $(m-1)$-dimensional homology group. Therefore, by the Hurewicz and Whitehead theorems, there are (homology and hence) weak homotopy equivalences of the form

$$
\bigvee_{k \in K} S_{k}^{m-1} \longrightarrow \widetilde{\dot{M}}
$$

and similarly

$$
\bigvee_{k \in K} S_{k}^{m-1} \vee \bigvee_{j=1}^{r-1} \bigvee_{g_{j} \in G} S_{j, g_{j}}^{m-1} \longrightarrow \stackrel{\dot{C}}{ }
$$

Thus the domain of $c$ is essentially the reduced $|p|$-dimensional homotopy group of the big wedge of spheres in 5.7; its Hilton decomposition (cf. [Hi]) is given by basic Whitehead products which for each $j=1, \ldots, r-1$ must involve a sphere $S_{j, g_{j}}^{m-1}$ for some covering transformation $g_{j} \in G$ (compare 5.4). But since $|p| \leq r(m-2)$, the remaining spheres $S_{k}^{m-1}$ cannot occur at all. Therefore $c$ just has the domain $\pi_{|p|}^{\prime}\left(\vee \vee S_{j, g_{j}}^{m-1}\right)$ and is seen to be an isomorphism.

Example 5.8 (Spherical space forms). Let $G$ be a finite group acting freely and smoothly on $S^{m}$. Then for $\kappa$-Brunnian link maps into the punctured orbit manifold $M=\left(S^{m} / G\right)-\left\{x^{\circ}\right\}$ the $\kappa$ - and $\widetilde{\mu}$-invariants are equally strong. In particular, every embedded codimension-2 link $f: \coprod^{r} S^{m-2} \hookrightarrow M$ is at least $\kappa_{M}$-trivial whenever $m \geq 4$; indeed, by a deep theorem of A. Bartels [B] all its liftings $\widetilde{f}^{g}$ (cf. 2.16) are link homotopy trivial in $S^{m}$.

In the case of punctured projective space $M=\mathbb{R} P^{m}-\left\{x^{\circ}\right\}$, the claim of theorem 5.6 can be deduced directly at the various steps of the construction of $\widetilde{\mu}$. 
Indeed, $M-\left\{y_{1}^{\circ}, \ldots, y_{r-1}^{\circ}\right\} \sim \mathbb{R} P^{m-1} \vee \bigvee^{r-1} S_{j}^{m-1}$; moreover, $\oplus \widetilde{c}^{g}$ (cf. 2.6) is just induced by the obvious collapsing maps

$$
\widetilde{M}-\bigcup_{j=1}^{r-1} p^{-1}\left\{y_{j}^{\circ}\right\} \sim S^{m-1} \vee \bigvee S_{j,+}^{m-1} \vee \bigvee S_{j,-}^{m-1} \longrightarrow \bigvee S_{j}^{m-1}
$$

and is therefore bijective on the reduced $|p|$-dimensional homotopy groups.

Next we observe that the assumption of high connectivity in theorem 5.6 cannot be dropped (compare also 4.11).

Example 5.9 (Higher dimensional Borromean links). Assume $1 \leq n \leq$ $m-2$. Let $S^{n} \subset \mathbb{R}^{n+1}$ be included in $S^{m-1} \subset \mathbb{R}^{m}$ in the standard fashion. Identify a tubular neighbourhood of $S^{n}$ in $\mathbb{R}^{m}$ with $S^{n} \times \mathbb{R}^{m-n}$. Also let $B_{2} \subset B_{1} \subset S^{m-1}$ be suitably embedded small compact $(m-1)$-balls whose center lies in $S^{n}$ and such that $B_{2} \subset \stackrel{\circ}{B}_{1}$.

Now define a link

$$
f=f_{1} \amalg f_{2}: S^{m-2} \amalg S^{n} \hookrightarrow M:=S^{n} \times \mathbb{R}^{m-n}
$$

as follows. $f_{1}$ is the inclusion of $\partial B_{1} \cong S^{m-2}$. $f_{2}$ is obtained by "doubling" the $n$-ball $D=S^{n}-\left(S^{n} \cap \stackrel{\circ}{B}_{2}\right)$ : push $D$ in $\mathbb{R}^{n+1}$ a little towards (and away from, resp.) the origin to obtain two embedded balls $D_{-}$and $D_{+}$which meet along their boundaries and together constitute the $n$-sphere $f_{2}\left(S^{n}\right)$.

Figure 5.10. The links $f$ and $f^{(1)}$

Clearly $f$ is homotopy (and even isotopy) trivial in $\mathbb{R}^{m}$ : deform first $D_{-}$ towards the center of $B_{2}$ and then $f_{1}$ towards the origin. In particular $\mu_{M}(f)=$ $\mu_{\mathbb{R}^{m}}(f)=0$ (compare 2.14).

On the other hand, $f$ is not $\kappa_{M}$-trivial in $M=S^{n} \times \mathbb{R}^{m-n}$. Indeed, a nulhomotopy of the product map $\widehat{f}^{M}$ in $\widetilde{C}_{2}(M)$ would induce a nulhomotopy of the product map $\widehat{f^{(1)}}$ of

$$
f^{(1)}:=e_{z} \amalg f: S^{m-n-1} \amalg S^{m-2} \amalg S^{n} \longrightarrow \mathbb{R}^{m}
$$

(where $e_{z}$ is a large meridian of $S^{n} \subset \mathbb{R}^{m}$ at some suitable point $z \in S^{n}$, cf. figure 5.10). But $\widehat{f^{(1)}}$ is projectable and therefore $\mu_{\mathbb{R}^{m}}\left(f^{(1)}\right)$ equals the linking 
number \pm 1 of an equator in $S^{n}$ with a pair of antipodal points (by [K 5], theorem 5.2 and the arguments in the proof of theorem 6.1).

If $n=1, \widetilde{M} \cong \mathbb{R}^{m}$ and theorem 5.6 applies: the nontriviality of $\kappa_{M}(f)$ must be detected by $\widetilde{\mu}_{M}(f)$ (though not by $\mu_{M}(f)$ ). (Also note that in the special case $m=3 \quad f^{(1)}$ is the classical Borromean link).

If $2 \leq n \leq m-2, \widetilde{M} \sim S^{n}$ is not $(m-2)$-connected and $\kappa_{M}(f)$ is strictly stronger than $\widetilde{\mu}_{M}(f)=\mu_{M}(f)=0$.

For a systematic comparison of $\kappa$ - and $\mu$-invariants in $S^{n} \times \mathbb{R}^{m-n}$ see [K 6$]$.

\section{$\S 6$. The classical dimension setting}

Throughout this section we assume the manifold $M$ to be 3-dimensional and oriented. We will consider link maps of the form

$$
f=\coprod_{j=1}^{r} f_{j}: \coprod_{j=1}^{r} S^{1} \longrightarrow M
$$

and reinterpret results of J. Milnor and U. Dahlmeier to show that in many significant cases the link homotopy class $[f]$ of $f$ is fully characterized by $\kappa_{M}(f)$ or even by the invariant $\widetilde{\mu}_{M}(f)$ which lies in (a quotient of) the group ring $J\left(G^{r-1} \times \Sigma_{r-2}\right)$ (since $\pi_{0}^{S}=\mathbb{Z}$ ).

In order to construct an inverse of $\widetilde{\mu}_{M}$ along the lines of Milnor's work [M] and our $\S 4$, pick a compact 3-ball $B \subset M$ which contains the base points $y_{1}^{\circ}, \ldots, y_{r-1}^{\circ}$ in its interior $\stackrel{\circ}{B}$ and $y_{r}^{\circ}$ in $\partial B$. Let $L=\ell_{1} \amalg \cdots \amalg \ell_{r-1} \subset \stackrel{\circ}{B}$ be a trivial (base point preserving) link consisting of the standard circles in $r-1$ parallel planes and let $a_{1}, \ldots, a_{r-1}$ denote meridians (in $B$ and starting at $y_{r}^{\circ}$ ) of $L$ (compare [M 1], p. 181, and our figure 4.3). Now, given an element

$$
\left(g=\left(g_{1}, \ldots, g_{r-1}\right), \gamma=\left(i_{1}, \ldots, i_{r-2}\right)\right) \in G^{r-1} \times \Sigma_{r-2},
$$

define $\varphi_{0}, \ldots, \varphi_{r-2} \in \pi_{1}\left(M-\stackrel{\circ}{B} ; y_{r}^{\circ}\right) \cong G$ by $\varphi_{0}=g_{i_{1}}, \quad \varphi_{r-2}=g_{i_{r-2}}^{-1} g_{r-1}$ and

$$
\varphi_{j}=g_{i_{j}}^{-1} g_{i_{j+1}} \quad \text { for } j=1, \ldots, r-3
$$

furthermore, just as in [M 1], p. 185, the resulting element

$$
s(g, \gamma)=\varphi_{0}\left(a_{i_{1}}-1\right) \varphi_{1}\left(a_{i_{2}}-1\right) \varphi_{2} \ldots \varphi_{r-3}\left(a_{i_{r-2}}-1\right) \varphi_{r-2}
$$

in the group ring $J\left(\pi_{1}(M-L)\right)$ gives rise to some product $a_{r-1}^{s(g, \gamma)} \in \pi_{1}(M-L)$ of conjugates of the meridian $a_{r-1}$ (at this stage such a product still depends on the chosen order of its factors). By extension, every element $\chi \in J\left(G^{r-1} \times \Sigma_{r-2}\right)$ yields $a_{r-1}^{s(\chi)} \in \pi_{1}(M-L)$ and hence a link homotopy class

$$
e(\chi)=\left[\ell_{1} \amalg \cdots \amalg \ell_{r-1} \amalg a_{r-1}^{s(\chi)}\right] .
$$

The following result is essentially due to J. Milnor. 
Theorem 6.3. The construction above determines a welldefined map e from the group ring of $G^{r-1} \times \Sigma_{r-2}$ onto the set of base point preserving link homotopy classes of all homotopy Brunnian link maps $f: \coprod^{r} S^{1} \longrightarrow M$.

Proof. Milnor's basic link homotopy (cf. [M 1], p. 183) allows to eliminate any commutator of conjugates of $a_{r-1}$ in the product $a_{r-1}^{s(\chi)}$; hence $e(\chi)$ does not depend on how these factors are ordered.

Next let the generic base point preserving link map $f$ be homotopy Brunnian. Then there exists a base point preserving link homotopy from $f_{1} \amalg \cdots \amalg f_{r-1}$ to $L$ which can be decomposed into ambient isotopies on one hand and, on the other hand, local crossing changes of arcs from the same link component; clearly, this can be extended to a link homotopy from $f$ to a link map of the form $L \amalg f_{r}^{\prime}$ (compare e.g. [L], p. 363-364, or [D], p. 2).

Now use the results and terminology of Milnor [M 1]. By his corollary 1 (p. 182), the class of $f_{r}^{\prime}$ in the link group $\mathcal{G}(L)$ lies already in the kernel $\mathcal{A}_{j}, j=1, \ldots, r-1$. It then follows from theorems 5 and 7 and again corollary 1 that $\left[f_{r}^{\prime}\right]$ can be expressed in precisely one way in the form $\left[a_{r-1}\right]^{s}$ where $s=\Sigma \pm s\left(g_{k} \gamma_{k}\right)$ is a sum of "canonical words" as in 6.2 above. Milnor's theorem 3 (and its proof) now implies our surjectivity claim.

Theorem 6.4. Up to a fixed sign $\widetilde{\mu}_{M^{\circ}} e$ is the identity on the group ring of $G^{r-1} \times$ $\Sigma_{r-2}$. Hence $\pm \widetilde{\mu}_{M}$ defines a bijection (inverse to $e$ ) from the base point preserving link homotopy set of classical homotopy Brunnian link maps in $M$ onto $J\left(G^{r-1} \times\right.$ $\left.\Sigma_{r-2}\right)$.

Proof. Given $\chi \in J\left(G^{r-1} \times \Sigma_{r-2}\right)$ and $\left(g^{\prime}, \gamma^{\prime}\right) \in G^{r-1} \times \Sigma_{r-2}$, the $\gamma^{\prime}$-part of $\widetilde{\mu}_{M, g^{\prime}}(e(\chi))$ is the $\mu_{\widetilde{M}, \gamma^{\prime}}$-invariant of the $g^{\prime}$-lifting $\widetilde{e(\chi)} g^{\prime}$ of $e(\chi)$ (see corollary 2.16). Now this link map in $\widetilde{M}$ consists of the small unlinked circles $g_{1}^{\prime} \circ \tilde{\ell}_{1} \amalg \cdots \amalg g_{r-1}^{\prime} \circ \widetilde{\ell}_{r-1}$ and of the standard lifting $\tilde{f}_{r}$ of $f_{r}=a_{r-1}^{s(\chi)}$. Thus we can apply proposition 2.20 and obtain

$$
\widetilde{\mu}_{M, g^{\prime}, \gamma^{\prime}}(e(\chi))=\mu_{\widetilde{M}, \gamma^{\prime}}\left(\widetilde{e(\chi)^{g}}\right)= \pm h_{\gamma^{\prime}}(b) ;
$$

here $b$ is the "link map" $\amalg N_{j} \subset \mathbb{R}$ formed by those isolated points in the domain of $\tilde{f}_{r}$ where $\tilde{f}_{r}$ intersects a small ball bounded by $g_{j}^{\prime} \circ \widetilde{\ell}_{j}, j=1, \ldots, r-1$ (see 2.18). If a summand $(g, \gamma) \in G^{r-1} \times \Sigma_{r-2}$ of $\chi$ and the corresponding summand $s(g, \gamma)$ of $s(\chi)$ (see 6.2) is to contribute to $h_{\gamma^{\prime}}(b)$ then $\gamma$ must equal $\gamma^{\prime}$ (compare [K 5], top of p. 315). Similarly, only the "principal part"

$$
s=\varphi_{0} a_{i_{1}} \varphi_{1} a_{i_{2}} \ldots a_{i_{r-2}} \varphi_{r-2}
$$

of $s(g, \gamma)$ (compare 6.2) is relevant for the calculation of $h_{\gamma^{\prime}}(b)$. Now note that $s$ lifts to a path $\widetilde{s}$ in $\widetilde{M}$ which first proceeds from $\widetilde{y}_{r}^{\circ}$ to $\varphi_{0}\left(\widetilde{y}_{r}^{\circ}\right)=g_{i_{1}}\left(\widetilde{y}_{r}^{\circ}\right)$, then traverses the meridian $g_{i_{1}}\left(\widetilde{a}_{i_{1}}\right)$, then goes on to $\varphi_{0} \varphi_{1}\left(\widetilde{y}_{r}^{\circ}\right)=g_{i_{2}}\left(\widetilde{y}_{r}^{\circ}\right)$, traverses $g_{i_{2}}\left(\widetilde{a}_{i_{2}}\right)$ etc. until it reaches $\varphi_{0} \varphi_{1} \ldots \varphi_{r-2}\left(\widetilde{y}_{r}^{\circ}\right)=g_{r-1}\left(\widetilde{y}_{r}^{\circ}\right)$ (compare 6.1). Therefore $a_{r-1}^{s}=s a_{r-1} s^{-1}$ (cf. [M 1], p. 185) lifts to $\widetilde{s}\left(g_{r-1}\left(\widetilde{a}_{r-1}\right)\right) \widetilde{s}^{-1}$ and contributes to the intersection $N_{j}$ precisely if the meridian $g_{j}\left(\widetilde{a}_{j}\right)$ links the component $g_{j}^{\prime} \circ \widetilde{\ell}_{j}$ 
of $\widetilde{e(\chi)} g^{\prime}$, i.e. if $g_{j}=g_{j}^{\prime}, j=1, \ldots, r-1$. We conclude that, up to a sign, $h_{\gamma^{\prime}}(b)$ is just the coefficient of $\left(g^{\prime}, \gamma^{\prime}\right)$ in $\chi$.

By induction (over the number of components in sublinks) the conclusion of theorem 6.4 allows to re-express its assumption: a classical link map in $M$ is homotopy Brunnian if and only if it is $\kappa_{M}$-Brunnian (cf. 1.7). Similarly we obtain

Corollary 6.5. For any link map $f=\coprod f_{j}: \coprod^{r} S^{1} \longrightarrow M^{3}$ the following conditions are equivalent:

(i) $f$ is link homotopically trivial;

(ii) $\kappa_{M}(f)$ is trivial; and

(iii) each component map $f_{j}$ is nulhomotopic in the usual sense, $1 \leq j \leq r$, and all (successively defined) $\widetilde{\mu}_{M}$-invariants of all sub-link maps of $f$ (proper or not) vanish.

(Note that for each of these statements the base point preserving and base point free versions are also equivalent.)

For example, if a classical link which lies in a 3-ball $B \subset M$ is link homotopically trivial in $M$ then also already in $B$; in other words, the extra space $M-B$ is not needed for the nulhomotopy. This consequence of the compatibility of the $\mu$ invariants in $B$ and in $M$ was pointed out to me by G.S. Li; in case $M$ is simply connected, it follows also from $[\mathrm{H}]$.

Corollary 6.6. Both in the base point free and base point preserving setting the $\kappa_{M}$-invariant characterises $\kappa_{M}$-Brunnian classical link maps completely up to link homotopy.

This was conjectured by U. Kaiser.

Question 6.7. When does $\kappa_{M}$ classify all classical link maps?

The following sample answer is a consequence of the complete classification results in $[\mathrm{D}]$.

Proposition 6.8 (U. Dahlmeier). If $M=S^{1} \times \mathbb{R}^{2}$ or $M=\mathbb{R} P^{3}-\{$ point $\}$, then $\kappa_{M}$ characterises arbitrary classical link maps with two components completely up to base point free link homotopy.

Proof. Let $f=f_{1} \amalg f_{2}$ and $f^{\prime}=f_{1}^{\prime} \amalg f_{2}^{\prime}$ be link maps $S^{1} \amalg S^{1} \longrightarrow M$ with equal $\kappa$-invariants.

If $M=S^{1} \times \mathbb{R}^{2}$, the winding numbers of $f_{j}$ and $f_{j}^{\prime}$ agree, $j=1,2$, and so does their greatest common denominator $d$. Now lift $f$ and $f^{\prime}$ (as well as the homotopy between the product maps $\widehat{f}$ and $\widehat{f}^{\prime}$ ) to link maps $\widetilde{f}$ and $\widetilde{f}^{\prime}$ in the connected d-fold covering space $\widetilde{M}_{d}$ of $S^{1} \times \mathbb{R}^{2}$ in such a way that $\widehat{\widetilde{f}} \sim \widehat{\widetilde{f^{\prime}}}$. Then the linking numbers of $\widetilde{f}$ and $\widetilde{f}^{\prime}$ in $\widetilde{M}_{d} \subset \mathbb{R}^{3}$ agree, and similarly for $\widetilde{f}_{1} \amalg g \widetilde{f}_{2}$ and $\widetilde{f}_{1}^{\prime} \amalg g \widetilde{f}_{2}^{\prime}$, where $g$ is any covering transformation. But according to theorem I in [D], these linking numbers determine $f$ and $f^{\prime}$ up to link homotopy.

If $M$ is a punctured projective 3-space, again the winding numbers of $f_{j}$ and $f_{j}^{\prime}$ agree, and so do the linking numbers in the sense of Seifert-Threlfall (which by 
theorem II in [D] classify the link maps $f$ and $f^{\prime}$ when they are not $\kappa$-Brunnian). Indeed, these linking numbers are suitably compatible both with precomposing $f, f^{\prime}$ by degree -2 selfmaps of $S^{1}$ and with liftings, and so are homotopies of the product $\operatorname{maps} \widehat{f}, \widehat{f}^{\prime}$.

\section{REFERENCES}

[B] A. Bartels, Link homotopy in codimension 2 (1999), Doctoral dissertation, UC San Diego.

[D] U. Dahlmeier, Verkettungshomotopien in Mannigfaltigkeiten (1993), Doctoral dissertation, Siegen.

[FN] E. Fadell and L. Neuwirth, Configuration spaces, Math. Scand. 10 (1962), 111-118.

[FR] R.A. Fenn and D. Rolfsen, Spheres may link homotopically in 4-space, J. London Math. Soc. 34 (1986), no. 2, 177-184.

[H] N. Habegger, Link homotopy in simply connected 3-manifolds, 1993 Georgia Top. Conf. Proceedings (1995).

[HK] N. Habegger and U. Kaiser, Link homotopy in the 2-metastable range, Topology 37 (1998), no. 1, 75-94.

[Hac] D. Hacon, Embeddings of $S^{p}$ in $S^{1} \times S^{q}$ in the metastable range, Topology 7 (1968), 1-10.

[Hi] P. Hilton, On the homotopy groups of the union of spheres, J. of the London Math. Soc. 30 (1955), 154-172.

[Ka] U. Kaiser, Link theory in manifolds, Lect. Notes in Math. 1669 (1997), Springer.

[Ki] P. Kirk, Link homotopy with one codimension 2 component, Trans. Am. Math. Soc. 319 (1990), 663-688.

[K 1] U. Koschorke, Link maps and the geometry of their invariants, Manuscr. math. 61 (1988), 383-415.

[K 2] On link maps and their homotopy classification, Math. Ann. 286 (1990), 753-782.

[K 3] — Link maps with many components, Topology 30 (1991), 267-282.

[K 4] Homotopy, concordance and bordism of link maps, Global analysis in modern mathematics, Publish or Perish, Houston, Texas, 1993, pp. 283-299.

[K 5] - A generalization of Milnor's $\mu$-invariants to higher-dimensional link maps, Topology 36 (1997), no. 2, 301-324.

[K 6] , Link homotopy in $S^{q} \times \mathbb{R}^{m-q}$ and higher order $\mu$-invariants, in preparation, Siegen (cf. also talk at ICM 1998 in Berlin).

[L] J. Levine, An approach to homotopy classification of links, Trans. AMS 306 (1988), no. 1, $361-387$.

[M 1] J. Milnor, Link groups, Ann. of Math. 59 (1954), 177-195.

[M 2] _ Differential topology, Mimeographed notes (taken by J. Munkres) of lectures at Princeton University, Fall 1958.

[Mu] J. Munkres, Elementary differential topology, Ann. of Math. Studies 54 (1966), Princeton Univ. Press.

[P] D. Puppe, Homotopiemengen und ihre induzierten Abbildungen, Math. Z. 69 (1958), 299344.

[T] P. Teichner, Symmetric surgery and boundary link maps (1997), Preprint, San Diego.

Universität Siegen, Emmy-Noether-Campus, D-57068 Siegen, Germany

E-mail address: koschorke@mathematik.uni-siegen.de 